\title{
STATIC KLEIN-GORDON-MAXWELL-PROCA SYSTEMS IN 4-DIMENSIONAL CLOSED MANIFOLDS
}

\author{
EMMANUEL HEBEY AND TRONG TUONG TRUONG
}

\begin{abstract}
We prove existence and uniform bounds for critical static KleinGordon-Maxwell-Proca systems in the case of 4-dimensional closed Riemannian manifolds.
\end{abstract}

Static Klein-Gordon-Maxwell-Proca systems are massive versions of the electrostatic Klein-Gordon-Maxwell Systems. The vector field in these systems inherits a mass and is governed by the Proca action which generalizes that of Maxwell. Klein-Gordon-Maxwell systems are intended to provide a dualistic model for the description of the interaction between a charged relativistic matter scalar field and the electromagnetic field that it generates. The electromagnetic field is both generated by and drives the particle field. In the electrostatic form of the Klein-GordonMaxwell systems, looking for standing waves $u e^{i \omega t}$, the matter field is characterized by the property that $u$, together with a gauge potential $v$, solve the electrostatic Klein-Gordon-Maxwell systems (0.3) with $m_{1}=0$. In the case of a closed manifold we discuss here the two equations in (0.3) are independent one of another when $m_{1}=0$ and the system reduces to the sole Schrödinger equation. The Proca formalism, for $m_{1}>0$, leads to a deeper phenomenon and is more appropriate to the closed case. The particle in this model interacts via the minimum coupling rule

$$
\partial_{t} \rightarrow \partial_{t}+i q \varphi \text { and } \nabla \rightarrow \nabla-i q A
$$

with an external massive vector field $(\varphi, A)$ which is governed by the Maxwell-Proca Lagrangian. The Proca action is a gauge-fixed version of the Stueckelberg action in the Higgs mechanism (see Goldhaber and Nieto [26, and Ruegg and Ruiz-Altaba [43]). In the Proca formalism, developped under the influence of de Broglie, the photon inherits a nonzero mass. This issue is of considerable importance and intensively studied in modern physics (see for instance Adelberger, Dvali and Gruzinov [1], Byrne [13, Goldhaber and Nieto [25, 26], Luo and Tu [38, Luo, Gillies and Tu [37. and the references in these papers). When $n=3$, the KGMP equations consist in the nonlinear Klein-Gordon matter equation, the charge continuity equation and the massive modified Maxwell equations in SI units, which are hereafter explicitly written down:

$$
\begin{aligned}
& \nabla . E=\rho / \varepsilon_{0}-\mu^{2} \varphi \\
& \nabla \times H=\mu_{0}\left(J+\varepsilon_{0} \frac{\partial E}{\partial t}\right)-\mu^{2} A, \\
& \nabla \times E+\frac{\partial H}{\partial t}=0 \text { and } \nabla \cdot H=0 .
\end{aligned}
$$

To appear in J. Reine Angew. Math. 
These massive Maxwell equations, as modified to Proca form, appear to have been first written in modern format by Schrödinger [46. The Proca formalism a priori breaks Gauge invariance. Gauge invariance can be restaured by the Stueckelberg trick, as pointed out by Pauli [41, and then by the Higgs mechanism. We refer to Goldhaber and Nieto [26, Luo, Gillies and Tu [37, and Ruegg and Ruiz-Altaba 43 for very complete references on the Proca approach.

In what follows we let $(M, g)$ be a smooth compact 3,4-dimensional Riemannian manifold. We let also $2^{\star}=\frac{2 n}{n-2}$ be the critical Sobolev exponent, where $n$ is the dimension of $M$. Given real numbers $q>0, m_{0}, m_{1}>0, \omega \in\left(-m_{0}, m_{0}\right)$, and $p \in\left(2,2^{\star}\right]$, the derivation of the Klein-Gordon-Maxwell-Proca system we investigate in this paper is written as

$$
\left\{\begin{array}{l}
\Delta_{g} u+m_{0}^{2} u=u^{p-1}+\omega^{2}(q v-1)^{2} u \\
\Delta_{g} v+\left(m_{1}^{2}+q^{2} u^{2}\right) v=q u^{2}
\end{array}\right.
$$

where $\Delta_{g}=-\operatorname{div}_{g} \nabla$ is the Laplace-Beltrami operator. The system (0.3) corresponds to looking for standing waves $u e^{i \omega t}$ for the full KGMP system in the static case where the massive vector field $(\varphi, A)$ depends on the sole spatial variable. The system is energy critical when $n=3$ and $p=6$ and when $n=4$ and $p=4$. It is subcritical otherwise, namely when $n=3$ and $p \in(2,6)$ or $n=4$ and $p \in(2,4)$. In the above model, $m_{1}$ is a coupling constant which makes that the two equations in (0.3) are trully coupled ( $m_{1}$ is the Proca mass in the Maxwell-Proca formalism) while $m_{0}$ is the mass of the particle, $q$ is the charge of the particle, $u$ is the amplitude in the writing of the particle, $\omega$ is its temporal frequency (referred to as the phase in the sequel), and $v$ is the electric potential.

Let $S_{g}$ stand for the scalar curvature of $g$, and $\mathcal{S}_{p}(\omega)$ be the set consisting of the positive smooth solutions $\mathcal{U}=(u, v)$ of $(0.3)$ with phase $\omega$ and nonlinear term $u^{p-1}$. Namely,

$$
\mathcal{S}_{p}(\omega)=\{(u, v) \text { smooth s.t. } u>0, v>0 \text {, and }(u, v) \text { solve }[\underline{0.3)}\} .
$$

Given $\omega \in\left[0, m_{0}\right)$, we let

$$
K_{0}(\omega)=\left(-m_{0},-\omega\right] \bigcup\left[\omega, m_{0}\right) .
$$

When $\omega=0, K_{0}(0)=\left(-m_{0}, m_{0}\right)$ is the full admissible phase range. For $\theta \in(0,1)$, and $\mathcal{U}=(u, v)$, we let $\|\mathcal{U}\|_{C^{2, \theta}}=\|u\|_{C^{2, \theta}}+\|v\|_{C^{2, \theta}}$. By a MPT solution we mean a solution with a strong mountain pass type structure. The following result was proved in Druet and Hebey [20.

Theorem 0.1 (The 3-dimensional case - Druet and Hebey [20]). Let $(M, g)$ be a smooth compact 3 -dimensional Riemannian manifold $m_{0}, m_{1}>0, \omega \in\left(-m_{0}, m_{0}\right)$, and $p \in(2,6]$. When $p=6$ assume

$$
m_{0}^{2}<\omega^{2}+\frac{1}{8} S_{g}(x)
$$

for all $x \in M$. Then (0.3) possesses a smooth positive MPT solution. Moreover, for any $p \in(2,6)$, and any $\theta \in(0,1)$, there exists $C>0$ such that for any $\omega^{\prime} \in K_{0}(0)$, and any $\mathcal{U} \in \mathcal{S}_{p}\left(\omega^{\prime}\right)$, $\|\mathcal{U}\|_{C^{2, \theta}} \leq C$, where $\mathcal{S}_{p}\left(\omega^{\prime}\right)$ is as in (0.4) and $K_{0}(0)$ is as in (0.5). Assuming again (0.6), there also holds that for any $\theta \in(0,1),\|\mathcal{U}\|_{C^{2, \theta}} \leq C$ for all $\mathcal{U} \in \mathcal{S}_{6}\left(\omega^{\prime}\right)$ and all $\omega^{\prime} \in K_{0}(\omega)$, where $C>0$ does not depend on $\omega^{\prime}$ and $\mathcal{U}$. 
This result exhibits phase compensation in the 3 -dimensional case. We aim in this paper in proving that a similar phenomenon holds true when $n=4$. In this dimension the second equation in (0.3) becomes critical and this leads to serious difficulties. We prove below the existence of smooth positive MPT solutions and the existence of uniform bounds for (0.3) in the subcritical cases $p \in(2,4)$ without any conditions, and in the critical case $p=4$ assuming that the mass potential, balanced by the phase, is smaller than the geometric threshold potential of the conformal Laplacian. In doing so we prove that phase compensation still holds true for our systems when $n=4$. Our result, in the subcritical case, is as follows.

Theorem 0.2 (The subcritical 4-dimensional case). Let $(M, g)$ be a smooth compact 4-dimensional Riemannian manifold, $q>0, m_{0}, m_{1}>0, \omega \in\left(-m_{0}, m_{0}\right)$, and $p \in(2,4)$. Then (0.3) possesses a smooth positive MPT solution. Moreover, for any $\theta \in(0,1)$, there exists $C>0$ such that for any $\omega^{\prime} \in K_{0}(0)$, and any $\mathcal{U} \in \mathcal{S}_{p}\left(\omega^{\prime}\right)$, $\|\mathcal{U}\|_{C^{2, \theta}} \leq C$, where $\mathcal{S}_{p}\left(\omega^{\prime}\right)$ is as in (0.4) and $K_{0}(0)$ is as in (0.5).

In the critical case we prove the following result. The geometry of the ambiant inhomogeneous space, through the scalar curvature of $g$, comes to play a role as in the 3-dimensional case. However, the result now turns out to be local in its existence part.

Theorem 0.3 (The critical 4-dimensional case). Let $(M, g)$ be a smooth compact 4-dimensional Riemannian manifold, $q>0, m_{0}, m_{1}>0, \omega \in\left(-m_{0}, m_{0}\right)$, and $p=4$. Assume

$$
m_{0}^{2}<\omega^{2}+\frac{1}{6} S_{g}(x)
$$

for some $x \in M$. Then (0.3) possesses a smooth positive MPT solution. Assuming that (0.7) holds true for all $x \in M$ there also holds that for any $\theta \in(0,1),\|\mathcal{U}\|_{C^{2, \theta}} \leq$ $C$ for all $\mathcal{U} \in \mathcal{S}_{4}\left(\omega^{\prime}\right)$ and all $\omega^{\prime} \in K_{0}(\omega)$, where $C>0$ does not depend on $\omega^{\prime}$ and $\mathcal{U}, \mathcal{S}_{4}\left(\omega^{\prime}\right)$ is as in (0.4), and $K_{0}(\omega)$ is as in (0.5).

There are two consequences to Theorem 0.3 . We list them in points (i)-(ii) below. In point (i) we illustrate the phase compensation effect associated with (0.3). There we always get existence and a priori bounds for all phases $\omega$ which are close to $m_{0}$. Point (ii) concerns the full range of phases when we assume $m_{0}$ is not too large.

(i) Phase compensation in the critical case - Assume $p=4$ and $S_{g}>0$ in $M$. Then there exists $\varepsilon>0$ such that for any $m_{0}-\varepsilon<|\omega|<m_{0}$, (0.3) possesses a smooth positive MPT solution. Moreover, for any $\theta \in(0,1)$, there exists $C>0$ such that $\|\mathcal{U}\|_{C^{2, \theta}} \leq C$ for all $\mathcal{U} \in \mathcal{S}_{4}(\omega)$ and all $m_{0}-\varepsilon<|\omega|<m_{0}$.

(iii) Full phase range in the critical case - Assume $p=4$ and $m_{0}^{2}<\frac{1}{6} S_{g}$ in $M$. For any $\omega \in\left(-m_{0}, m_{0}\right)$, (0.3) possesses a smooth positive MPT solution. Moreover, for any $\theta \in(0,1)$, there exists $C>0$ such that $\|\mathcal{U}\|_{C^{2, \theta}} \leq C$ for all $\mathcal{U} \in \mathcal{S}_{4}(\omega)$ and all $\omega \in\left(-m_{0}, m_{0}\right)$.

As an immediate consequence of the $C^{2, \theta}$-bounds in the above results we obtain phase stability for standing waves of the Klein-Gordon-Maxwell-Proca equations in electrostatic form. Standing waves for the Klein-Gordon-Maxwell-Proca equations in electrostatic form are written as $S=u e^{i \omega t}$ and they are coupled with a gauge potential $v$, where $(u, v)$ solves $(0.3)$. Roughly speaking, phase stability means that for any arbitrary sequence of standing waves $u_{\alpha} e^{i \omega_{\alpha} t}$, with gauge potentials $v_{\alpha}$, the convergence of the phases $\omega_{\alpha}$ in $\mathbb{R}$ implies the convergence of the amplitudes $u_{\alpha}$ 
and of the gauge potentials $v_{\alpha}$ in the $C^{2}$-topology. Phase stability prevents the existence of arbitrarily large amplitude standing waves.

High dimensional KGM systems in Coulomb gauge have been recently investigated by Rodnianski and Tao 42 and with special emphasis in $(1+4)$-dimensions by Klainerman and Tataru 29] and Selberg 47. Electrostatic KGM systems in the three dimensional case have been investigated by several authors. Possible references on the physics side are by Benci and Fortunato [5, 6, Long [35, Long and Stuart 36. Blowing-up solutions to the electrostatic Schrödinger-Maxwell system, a cousin of the electrostaic KGM type systems that we consider here, have been constructed in D'Aprile and Wei [2, 3].

We briefly discuss in Section 11 the physics relevance of (0.3). We prove our theorem in Sections 2 to 4. The existence part in the theorem is proved in Section 2. The $C^{2, \theta}$-bound in the subcritical case is established in Section 3 . The more delicate $C^{2, \theta}$-bound in the critical case is established in Sections 4 . The phase compensation phenomenon in the theorem holds true thanks to the 4-dimensional $\log$ effect $\mu^{2}=o\left(\mu^{2} \log \mu\right)$ as $\mu \rightarrow 0$.

\section{THE PHYSICS ORIGIN OF THE SYSTEM}

The Klein-Gordon-Maxwell-Proca system discussed in this work describes an interacting field theory model in theoretical physics. Most electromagnetic phenomena are described by conventional electrodynamics, which is a theory of the coupling of electromagnetic fields to matter fields. Of prime importance for particle physics is fermion electrodynamics in which matter is represented by spinor fields. However one may have also boson electrodynamics in which matter is described by integer spin or bosonic fields. The simplest one is of course the complex scalar field, describing spinless particles having electric charges $\pm q$. It gives rise to scalar electrodynamics, which describes in the non-relativistic limit the superconductivity of metals at very low temperatures. In the more general context of particle physics, a complex scalar field $\psi$ may serve to describe scalar mesons in nuclear matter interacting via a massive vector boson field $(\varphi, A)$.

The interaction in this model is described by the minimum substitution rule (0.1) in a nonlinear Klein-Gordon Lagrangian. As for the external massive vector field it is governed by the Maxwell-Proca Lagrangian. More precisely, assuming for short that the manifold is orientable, we define the Lagrangian densities $\mathcal{L}_{N K G}$ and $\mathcal{L}_{M P}$ of $\psi, \varphi$, and $A$ by

$$
\begin{aligned}
& \mathcal{L}_{N K G}(\psi, \varphi, A)=\frac{1}{2}\left|\left(\frac{\partial}{\partial t}+i q \varphi\right) \psi\right|^{2}-\frac{1}{2}|(\nabla-i q A) \psi|^{2}+\frac{m_{0}^{2}}{2}|\psi|^{2}-\frac{1}{p}|\psi|^{p}, \\
& \mathcal{L}_{M P}(\varphi, A)=\frac{1}{2}\left|\frac{\partial A}{\partial t}+\nabla \varphi\right|^{2}-\frac{1}{2}|\nabla \times A|^{2}+\frac{m_{1}^{2}}{2}|\varphi|^{2}-\frac{m_{1}^{2}}{2}|A|^{2},
\end{aligned}
$$

where $\nabla \times=\star d, \star$ is the Hodge dual, $\psi$ represents the matter complex scalar field, $m_{0}$ its mass, $q$ its charge, $(\varphi, A)$ the electromagnetic vector field, and $m_{1}$ its mass. It can be noted that $\|(\varphi, A)\|_{L}^{2}=|\varphi|^{2}-|A|^{2}$ is the square of the Lorentz norm of $(\varphi, A)$ with respect to the Lorentz metric $\operatorname{diag}(1,-1, \ldots,-1)$. The total action functional for $\psi, \phi$, and $A$ is then given by

$$
\mathcal{S}(\psi, \varphi, A)=\iint\left(\mathcal{L}_{N K G}+\mathcal{L}_{M P}\right) d v_{g} d t
$$


Writing $\psi$ in polar form as $\psi(x, t)=u(x, t) e^{i S(x, t)}$, taking the variation of $\mathcal{S}$ with respect to $u, S, \varphi$, and $A$, we get four equations which are written as

$$
\left\{\begin{array}{l}
\frac{\partial^{2} u}{\partial t^{2}}+\Delta_{g} u+m_{0}^{2} u=u^{p-1}+\left(\left(\frac{\partial S}{\partial t}+q \varphi\right)^{2}-|\nabla S-q A|^{2}\right) u \\
\frac{\partial}{\partial t}\left(\left(\frac{\partial S}{\partial t}+q \varphi\right) u^{2}\right)-\nabla \cdot\left((\nabla S-q A) u^{2}\right)=0 \\
-\nabla \cdot\left(\frac{\partial A}{\partial t}+\nabla \varphi\right)+m_{1}^{2} \varphi+q\left(\frac{\partial S}{\partial t}+q \varphi\right) u^{2}=0 \\
\bar{\Delta}_{g} A+\frac{\partial}{\partial t}\left(\frac{\partial A}{\partial t}+\nabla \varphi\right)+m_{1}^{2} A=q(\nabla S-q A) u^{2}
\end{array}\right.
$$

where $\Delta_{g}=-\operatorname{div}_{g} \nabla$ is the Laplace-Beltrami operator, $\bar{\Delta}_{g}=\delta d$ is half the Laplacian acting on forms, and $\delta$ is the codifferential. We refer to this system as a nonlinear Klein-Gordon-Maxwell-Proca system. When $n=3, \bar{\Delta}_{g} A=\nabla \times(\nabla \times A)$ and if we let

$$
\begin{aligned}
& E=-\left(\frac{\partial A}{\partial t}+\nabla \varphi\right), H=\nabla \times A, \\
& \rho=-\left(\frac{\partial S}{\partial t}+q \varphi\right) q u^{2}, \text { and } j=(\nabla S-q A) q u^{2},
\end{aligned}
$$

then the two last equations in (1.3) give rise to the first pair of the MaxwellProca equations (0.2) with $\epsilon_{0}=\mu_{0}=1$ (units are chosen such that $c=1$ ) and $\mu^{2}=m_{1}^{2}$, while the two first equations in (1.4) give rise to the second pair of the equations. The first equation in (1.3) gives rise to the nonlinear Klein-Gordon matter equation. The second equation in (1.3) gives rise to the charge continuity equation $\frac{\partial \rho}{\partial t}+\nabla \cdot j=0$ which, thanks to (0.2), is equivalent to the Lorentz condition $\nabla \cdot A+\frac{\partial \varphi}{\partial t}=0$.

We assume in what follows that $u(x, t)=u(x)$ does not depend on $t, S(x, t)=\omega t$ does not depend on $x$, and $\varphi(x, t)=\varphi(x), A(x, t)=A(x)$ do not depend on $t$. In other words, we look for standing waves solutions of (1.3) and assume that we are in the static case of the system where $(\varphi, A)$ depends on the sole spatial variable. By the fourth equation in (1.3) we then get that

$$
\bar{\Delta}_{g} A+\left(q^{2} u^{2}+m_{1}^{2}\right) A=0 .
$$

This clearly implies that, and is equivalent to, $A \equiv 0$ since

$$
\int\left(\bar{\Delta}_{g} A, A\right)=\int|d A|^{2} \text {. }
$$

As a remark, assuming that $A \equiv 0$, the Lorentz condition for the external Proca field $(\varphi, A)$ would make $\varphi$ dependent on the sole spatial variables. As for the second equation in (1.3) it reduces to $\frac{\partial^{2} S}{\partial t^{2}}=0$. It is automatically satisfied when $S(t)=\omega t$, and we are thus left with the first and third equations in (1.3). Letting $S=-\omega t$, and $\varphi=\omega v$, these equations are rewritten as

$$
\left\{\begin{array}{c}
\Delta_{g} u+m_{0}^{2} u=u^{p-1}+(q \varphi-\omega)^{2} u \\
\Delta_{g} \varphi+m_{1}^{2} \varphi+q(q \varphi-\omega) u^{2}=0 .
\end{array}\right.
$$

In particular, letting $\varphi=\omega v$, in (1.5), we recover our original system (0.3). In other words, our original system (0.3) corresponds to looking for standing waves solutions of the Klein-Gordon-Maxwell-Proca system (1.3) in static form. 


\section{Existence Theory}

We prove the existence part in Theorems 0.2 and 0.3 and look for solutions with a special variational structure. Formally, solutions of (0.3) are critical points of the functional $S$ defined by

$$
\begin{aligned}
S(u, v) & =\frac{1}{2} \int_{M}|\nabla u|^{2} d v_{g}-\frac{\omega^{2}}{2} \int_{M}|\nabla v|^{2} d v_{g}+\frac{m_{0}^{2}}{2} \int_{M} u^{2} d v_{g} \\
& -\frac{\omega^{2} m_{1}^{2}}{2} \int_{M} v^{2} d v_{g}-\frac{1}{p} \int_{M} u^{p} d v_{g}-\frac{\omega^{2}}{2} \int_{M} u^{2}(1-q v)^{2} d v_{g} .
\end{aligned}
$$

The functional $S$ is strongly indefinite because of the competition between $u$ and $v$. Following a very nice idea going back to Benci-Fortunato [5], we introduce the auxiliary functional $\Phi$ given by

$$
\Delta_{g} \Phi(u)+\left(m_{1}^{2}+q^{2} u^{2}\right) \Phi(u)=q u^{2},
$$

and then consider that $u$ in (0.3) can be seen as a critical point of

$$
\begin{aligned}
I_{p}(u) & =\frac{1}{2} \int_{M}|\nabla u|^{2} d v_{g}+\frac{m_{0}^{2}}{2} \int_{M} u^{2} d v_{g}-\frac{1}{p} \int_{M}\left(u^{+}\right)^{p} d v_{g} \\
& -\frac{\omega^{2}}{2} \int_{M}(1-q \Phi(u)) u^{2} d v_{g},
\end{aligned}
$$

where $u^{+}=\max (u, 0)$. We explicitly define MPT solutions to be solutions we obtain from $I_{p}$ by the mountain pass lemma from 0 to $u_{1}$ with $\left\|u_{1}\right\|_{L^{p}}^{p}$ being as large as we want with respect to $\left\|u_{1}\right\|_{H^{1}}^{2}$. Let $\Psi: H^{1}(M) \rightarrow \mathbb{R}$ be defined by

$$
\Psi(u)=\frac{1}{2} \int_{M}(1-q \Phi(u)) u^{2} d v_{g} .
$$

The following lemma establishes the existence and differentiability of $\Phi$, as well as the $C^{1}$-smoothness of $\Psi$. Equation (2.2) is critical when $n=4$ because of the term $u^{2} \Phi(u)$.

Lemma 2.1. Let $(M, g)$ be a smooth compact Riemannian 4-manifold and $q>0$. There exists $\Phi: H^{1}(M) \rightarrow H^{1}(M)$ such that (2.2) holds true and $0 \leq \Phi(u) \leq \frac{1}{q}$ for all $u \in H^{1}(M)$. Moreover, $\Phi$ is locally Lipschitz and differentiable. Its differential $D \Phi(u)=V_{u}$ at $u$ is given by

$$
\Delta_{g} V_{u}(\varphi)+\left(m_{1}^{2}+q^{2} u^{2}\right) V_{u}(\varphi)=2 q u(1-q \Phi(u)) \varphi
$$

for all $\varphi \in H^{1}(M)$. The functional $\Psi: H^{1}(M) \rightarrow \mathbb{R}$ defined in (2.4) is $C^{1}$ in $H^{1}(M)$ and

$$
D \Psi(u) \cdot(\varphi)=\int_{M}(1-q \Phi(u))^{2} u \varphi d v_{g}
$$

for all $u, \varphi \in H^{1}(M)$.

Proof of Lemma 2.1. We briefly sketch the proof. Let $u \in H^{1}$ and $H_{u}: H^{1} \rightarrow \mathbb{R}$ be defined by

$$
H_{u}(\varphi)=\int_{M}|\nabla \varphi|^{2} d v_{g}+\int_{M}\left(m_{1}^{2}+q^{2} u^{2}\right) \varphi^{2} d v_{g} .
$$

The functional is well defined since $H^{1} \subset L^{4}$. Letting $\Phi(0)=0$ we can assume that $u \not \equiv 0$. Let

$$
\mu=\inf _{u \in H^{1}, \int u^{2} \varphi=1} H_{u}(\varphi)
$$


By standard minimization arguments there exists $\varphi \in H^{1}(M)$ such that $\int_{M} u^{2} \varphi d v_{g}=$ 1 and $H_{u}(\varphi)=\mu$. In particular, $\mu>0$. Letting $\Phi(u)=\frac{q}{\mu} \varphi$ we get that $\Phi(u)$ solves (2.2) in $H^{1}$. It is easily seen that $\Phi(u)$ is unique. By the maximum principle, $\Phi(u) \geq 0$. Noting that

$$
\Delta_{g}\left(\frac{1}{q}-\Phi(u)\right)+\left(m_{1}^{2}+q^{2}\right) u^{2}\left(\frac{1}{q}-\Phi(u)\right) \geq 0
$$

it also follows from the maximum principle that $\Phi(u) \leq \frac{1}{q}$. Now we let $u, v \in$ $H^{1}(M)$. We have that

$$
\Delta_{g}(\Phi(v)-\Phi(u))+\left(m_{1}^{2}+q^{2}\right) u^{2}(\Phi(v)-\Phi(u))=q\left(v^{2}-u^{2}\right)(1-q \Phi(v)) .
$$

Multiplying the equation by $\Phi(v)-\Phi(u)$, integrating over $M$, and by the Sobolev emedding theorem, we get that

$$
\|\Phi(v)-\Phi(u)\|_{H^{1}} \leq C\left(\|u\|_{H^{1}}+\|v\|_{H^{1}}\right)\|v-u\|_{H^{1}} .
$$

In particular, $\Phi$ is locally Lipschitz continuous. We can prove the existence of $V_{u}(\varphi)$ in (2.5) as when proving the existence of $\Phi(u)$. Writing the equation satisfied by $\Phi(u+\varphi)-\Phi(u)-V_{u}(\varphi)$, multiplying the equation by $\Phi(u+\varphi)-\Phi(u)-V_{u}(\varphi)$ and integrating over $M$, we get that

$$
\left\|\Phi(u+\varphi)-\Phi(u)-V_{u}(\varphi)\right\|_{H^{1}} \leq C\|\varphi\|_{H^{1}}\left(\|\varphi\|_{H^{1}}+\|u\|_{H^{1}}\|\Phi(u+\varphi)-\Phi(u)\|_{H^{1}}\right)
$$

Then the differentiability of $\Phi$ follows from the continuity of $\Phi$. In particular, $\Psi$ is differentiable. By (2.2),

$$
\Psi(u)=\frac{1}{2} \int_{M}\left(|\nabla \Phi(u)|^{2}+m_{1}^{2} \Phi(u)^{2}\right) d v_{g}+\frac{1}{2} \int_{M}(1-q \Phi(u))^{2} u^{2} d v_{g},
$$

and we also have that $\frac{\partial H}{\partial \Phi}(u, \Phi(u))=0$, where $H(u, \Phi)=\frac{1}{2} H_{u}(\Phi)-q \int_{M} u^{2} \Phi d v_{g}$. Noting that

$$
\Psi(u)=H(u, \Phi(u))+\frac{1}{2} \int_{M} u^{2} d v_{g},
$$

we get that (2.6) holds true. The continuity of $D \Psi$ can be proved directly from (2.6) and the continuity of $\Phi$. This ends the proof of the lemma.

Now we prove the subcritical existence of Theorem 0.2 . We proceed by applying the mountain pass lemma to the functional $I_{p}$ in (2.3).

Proof of existence in Theorem 0.2. By Lemma 2.1, $I_{p}$ is $C^{1}$ in $H^{1}$. Let $u_{0} \in H^{1}$ such that $u_{0}^{+} \not \equiv 0$. There holds $I_{p}(0)=0$ and $I_{p}\left(t u_{0}\right) \rightarrow-\infty$ as $t \rightarrow+\infty$ since $p>2$. Since $0 \leq \Phi(u) \leq \frac{1}{q}$ for all $u$, we also have that

$$
\begin{aligned}
I_{p}(u) & \geq \frac{1}{2}\left(\int_{M}|\nabla u|^{2} d v_{g}+\left(m_{0}^{2}-\omega^{2}\right) \int_{M} u^{2} d v_{g}\right)-\frac{1}{p} \int_{M}|u|^{p} d v_{g} \\
& \geq C_{1}\|u\|_{H^{1}}^{2}-C_{2}\|u\|_{H^{1}}^{p}
\end{aligned}
$$

for all $u \in H^{1}$, where $C_{1}, C_{2}>0$ do not depend on $u$. In particular, there exist $\delta, C>0$ such that $I_{p}(u) \geq C$ for all $u \in H^{1}$ such that $\|u\|_{H^{1}}=\delta$. Let $T_{0}=T_{0}\left(u_{0}\right)$, $T_{0} \gg 1$, be such that $I_{p}\left(T_{0} u_{0}\right)<0$, and $c_{p}=c_{p}\left(u_{0}\right)$ be given by

$$
c_{p}=\inf _{P \in \mathcal{P}} \max _{u \in P} I_{p}(u),
$$

where $\mathcal{P}$ is the class of continuous paths joining 0 to $T_{0} u_{0}$. According to the above we can apply the mountain pass lemma and we get the existence of a sequence 
$\left(u_{\alpha}\right)_{\alpha}$ in $H^{1}$ such that $I_{p}\left(u_{\alpha}\right) \rightarrow c_{p}$ and $D I_{p}\left(u_{\alpha}\right) \rightarrow 0$ as $\alpha \rightarrow+\infty$. Writing that $I_{p}\left(u_{\alpha}\right)=c_{p}+o(1)$ and that $D I_{p}\left(u_{\alpha}\right) \cdot\left(u_{\alpha}\right)=o\left(\left\|u_{\alpha}\right\|_{H^{1}}\right)$, we get by Lemma 2.1 that

$$
\begin{aligned}
& \frac{1}{2} \int_{M}\left(\left|\nabla u_{\alpha}\right|^{2}+m_{0}^{2} u_{\alpha}^{2}\right) d v_{g} \\
& \quad=\frac{1}{p} \int_{M}\left(u_{\alpha}^{+}\right)^{p} d v_{g}+c_{p}+\frac{\omega^{2}}{2} \int_{M}\left(1-q \Phi\left(u_{\alpha}\right)\right) u_{\alpha}^{2} d v_{g}+o(1) \\
& \frac{1}{2} \int_{M}\left(\left|\nabla u_{\alpha}\right|^{2}+m_{0}^{2} u_{\alpha}^{2}\right) d v_{g} \\
& \quad=\frac{1}{2} \int_{M}\left(u_{\alpha}^{+}\right)^{p} d v_{g}+\frac{\omega^{2}}{2} \int_{M}\left(1-q \Phi\left(u_{\alpha}\right)\right)^{2} u_{\alpha}^{2} d v_{g}+o\left(\left\|u_{\alpha}\right\|_{H^{1}}\right)
\end{aligned}
$$

for all $\alpha$. Writing that $D I_{p}\left(u_{\alpha}\right) \cdot\left(u_{\alpha}^{-}\right)=o\left(\left\|u_{\alpha}^{-}\right\|_{H^{1}}\right)$ we get that $u_{\alpha}^{-} \rightarrow 0$ in $H^{1}$ as $\alpha \rightarrow+\infty$. By (2.9) we then get that $\left(u_{\alpha}\right)_{\alpha}$ is bounded in $H^{1}$. In particular, there exists $u_{p} \in H^{1}(M)$ such that, up to passing to a subsequence,

(i) $u_{\alpha} \rightarrow u_{p}$ weakly in $H^{1}$,

(ii) $u_{\alpha} \rightarrow u_{p}$ in $L^{p}$

and $u_{\alpha} \rightarrow u_{p}$ a.e. as $\alpha \rightarrow+\infty$. Substracting one equation to another in (2.9), letting $\alpha \rightarrow+\infty$, and since $c_{p} \neq 0$, we get that $u_{p} \neq \equiv 0$. Writing the equation satisfied by $\Phi\left(u_{\alpha}\right)-\Phi\left(u_{p}\right)$, multiplying the equation by $\Phi\left(u_{\alpha}\right)-\Phi\left(u_{p}\right)$ and integrating over $M$, we get that

$$
\Phi\left(u_{\alpha}\right) \rightarrow \Phi\left(u_{p}\right) \text { in } H^{1}
$$

as $\alpha \rightarrow+\infty$. Now we let $\varphi \in H^{1}$. There holds $D I_{p}\left(u_{\alpha}\right) \cdot(\varphi)=o(1)$. Hence, by Lemma 2.1

$$
\begin{aligned}
& \int_{M} \nabla u_{\alpha} \nabla \varphi d v_{g}+m_{0}^{2} \int_{M} u_{\alpha} \varphi d v_{g} \\
& =\int_{M}\left(u_{\alpha}^{+}\right)^{p-1} \varphi d v_{g}+\omega^{2} \int_{M}\left(1-q \Phi\left(u_{\alpha}\right)\right)^{2} u_{\alpha} \varphi d v_{g}+o(1) .
\end{aligned}
$$

Letting $\alpha \rightarrow+\infty$ in (2.11) we then get by (2.10) that

$$
\Delta_{g} u_{p}+m_{0}^{2} u_{p}=\left(u_{p}^{+}\right)^{p-1}+\omega^{2}\left(1-q \Phi\left(u_{p}\right)\right)^{2} u_{p}
$$

in $H^{1}$. Multiplying the equation by $u_{p}^{-}$and integrating over $M$, it follows that $u_{p}^{-} \equiv 0$. In particular, $u_{p} \geq 0, u_{p} \neq \equiv 0$, and

$$
\Delta_{g} u_{p}+m_{0}^{2} u_{p}=u_{p}^{p-1}+\omega^{2}\left(1-q \Phi\left(u_{p}\right)\right)^{2} u_{p}
$$

in $H^{1}$. By regularity results we get from (2.12) that $u_{p} \in H^{2, s}$ for all $s$. Then, by regularity results, $\Phi\left(u_{p}\right) \in H^{2, s}$ for all $s$. By the Sobolev embedding theorem, regularity theory, and the maximum principle, it follows that $u_{p}, \Phi\left(u_{p}\right) \in C^{2}(M)$ and that $u_{p}, \Phi\left(u_{p}\right)>0$ in $M$. Letting $u=u_{p}$ and $v=\Phi\left(u_{p}\right)$, this proves the existence part in Theorem 0.2 .

An additional information we obtain is that $u_{p}$ realizes $c_{p}$. Indeed, since $u_{p} \geq 0$, $u_{\alpha}^{-} \rightarrow 0$ in $H^{1}$, and $\Phi\left(u_{\alpha}\right) \rightarrow \Phi\left(u_{p}\right)$ in $H^{1}$, there holds that

$$
\begin{aligned}
& \int_{M}\left(u_{\alpha}^{+}\right)^{p} d v_{g} \rightarrow \int_{M} u_{p}^{p} d v_{g}, \text { and } \\
& \int_{M}\left(1-q \Phi\left(u_{\alpha}\right)\right)^{2} u_{\alpha}^{2} d v_{g} \rightarrow \int_{M}\left(1-q \Phi\left(u_{p}\right)\right)^{2} u_{p}^{2} d v_{g}
\end{aligned}
$$


as $\alpha \rightarrow+\infty$. The second equation in (2.9) together with (2.12) then give that

$$
\int_{M}\left|\nabla u_{\alpha}\right|^{2} d v_{g} \rightarrow \int_{M}\left|\nabla u_{p}\right|^{2} d v_{g}
$$

It follows that $u_{\alpha} \rightarrow u_{p}$ in $H^{1}$ as $\alpha \rightarrow+\infty$. By the first equation in (2.9) we then get that $I_{p}\left(u_{p}\right)=c_{p}$. In other words, $c_{p}$ is realized by $u_{p}$. Now, given $x_{0} \in M$ and $\rho_{0}>0$ small, sufficiently small, we define $u_{\varepsilon}$ by

$$
\left\{\begin{array}{l}
u_{\varepsilon}(x)=\frac{\varepsilon}{\varepsilon^{2}+r^{2}}-\frac{\varepsilon}{\varepsilon^{2}+\rho_{0}^{2}} \text { if } r \leq \rho_{0} \\
u_{\varepsilon}(x)=0 \text { if } r \geq \rho_{0}
\end{array}\right.
$$

where $r=d_{g}\left(x_{0}, x\right)$. Then, see Aubin 4, for any $\lambda \in \mathbb{R}$,

$$
J_{\lambda}\left(u_{\varepsilon}\right)=\frac{1}{K_{4}^{2}}\left(1+C\left(\frac{1}{6} S_{g}\left(x_{0}\right)-\lambda\right) \varepsilon^{2} \ln \varepsilon+o\left(\varepsilon^{2} \ln \varepsilon\right)\right),
$$

where

$$
J_{\lambda}\left(u_{\varepsilon}\right)=\frac{\int_{M}\left(\left|\nabla u_{\varepsilon}\right|^{2}+\lambda u_{\varepsilon}^{2}\right) d v_{g}}{\left(\int_{M} u_{\varepsilon}^{4} d v_{g}\right)^{1 / 2}},
$$

and $C>0$ is independent of $\alpha$. Also there holds

$$
\begin{aligned}
& \int_{M} u_{\varepsilon}^{4} d v_{g}=\int_{\mathbb{R}^{3}}\left(\frac{1}{1+|x|^{2}}\right)^{4} d x+o(1), \\
& \int_{M}\left|\nabla u_{\varepsilon}\right|^{2} d v_{g}=8 \int_{M} u_{\varepsilon}^{4} d v_{g}+o(1) .
\end{aligned}
$$

In what follows we prove the existence part of Theorem 0.3

Proof of existence in Theorem 0.3. As a preliminary remark, by standard arguments such as developed in Aubin [4] and Brézis and Nirenberg [12, we just need to prove that we can chose $u_{0} \in H^{1}, u_{0}^{+} \not \equiv 0$, such that

$$
\delta_{0} \leq c_{p} \leq \frac{1}{4 K_{4}^{4}}-\delta_{0}
$$

for all $p \in(4-\varepsilon, 4)$ and some $\varepsilon, \delta_{0}>0$, where $c_{p}=c_{p}\left(u_{0}\right)$ is as in (2.8). Now we assume that (0.7) holds true for some $x \in M$, in particular for $x \in M$ where $S_{g}$ is maximum. We let $x_{0} \in M$ be such that $S_{g}$ is maximum at $x_{0}$, and $\left(t_{\varepsilon}\right)_{\varepsilon}$ be any family of positive real numbers such that the $t_{\varepsilon}$ 's are bounded. The first estimate we prove is that

$$
\int_{M} \Phi\left(t_{\varepsilon} u_{\varepsilon}\right) u_{\varepsilon}^{2} d v_{g}=O\left(\varepsilon^{2}\right),
$$

where the $u_{\varepsilon}$ 's are as in (2.13). By definition,

$$
\Delta_{g} \Phi\left(t_{\varepsilon} u_{\varepsilon}\right)+\left(m_{1}^{2}+q^{2}\right) t_{\varepsilon}^{2} u_{\varepsilon}^{2} \Phi\left(t_{\varepsilon} u_{\varepsilon}\right)=q t_{\varepsilon}^{2} u_{\varepsilon}^{2} .
$$

Multiplying (2.18) by $\Phi\left(t_{\varepsilon} u_{\varepsilon}\right)$ and integrating over $M$ we get by Hölder's inequalities that

$$
\begin{aligned}
\left\|\Phi\left(t_{\varepsilon} u_{\varepsilon}\right)\right\|_{H^{1}}^{2} & =q t_{\varepsilon}^{2} \int_{M} u_{\varepsilon}^{2} \Phi\left(t_{\varepsilon} u_{\varepsilon}\right) d v_{g} \\
& \leq C\left(\int_{M} u_{\varepsilon}^{8 / 3} d v_{g}\right)^{3 / 4}\left\|\Phi\left(t_{\varepsilon} u_{\varepsilon}\right)\right\|_{L^{4}}
\end{aligned}
$$


and it follows from the Sobolev inequality that

$$
\left\|\Phi\left(t_{\varepsilon} u_{\varepsilon}\right)\right\|_{H^{1}} \leq C\left(\int_{M} u_{\varepsilon}^{8 / 3} d v_{g}\right)^{3 / 4} .
$$

Then, by (2.19),

$$
\begin{aligned}
\int_{M} \Phi\left(t_{\varepsilon} u_{\varepsilon}\right) u_{\varepsilon}^{2} d v_{g} & \leq C\left(\int_{M} u_{\varepsilon}^{8 / 3} d v_{g}\right)^{3 / 4}\left\|\Phi\left(t_{\varepsilon} u_{\varepsilon}\right)\right\|_{L^{4}} \\
& \leq C\left(\int_{M} u_{\varepsilon}^{8 / 3} d v_{g}\right)^{3 / 2}
\end{aligned}
$$

There holds,

$$
\begin{aligned}
\int_{M} u_{\varepsilon}^{8 / 3} d v_{g} & \leq \omega_{3} \int_{0}^{\rho_{0}}\left(\frac{\varepsilon}{\varepsilon^{2}+r^{2}}\right)^{8 / 3} r^{3} d r \\
& =\omega_{3} \varepsilon^{4 / 3} \int_{0}^{\rho_{0} / \varepsilon}\left(\frac{1}{1+r^{2}}\right)^{8 / 3} r^{3} d r \\
& =O\left(\varepsilon^{4 / 3}\right) .
\end{aligned}
$$

By (2.20) and (2.21), this proves (2.17). Let $\left(\varepsilon_{\alpha}\right)_{\alpha}$ be a sequence of positive real numbers such that $\varepsilon_{\alpha} \rightarrow 0$ as $\alpha \rightarrow+\infty, u_{\alpha}=u_{\varepsilon_{\alpha}}$, and $\mathcal{F}_{4}$ be the functional defined in $H^{1}$ by

$$
\mathcal{F}_{4}(u)=\frac{1}{2} \int_{M}|\nabla u|^{2} d v_{g}+\frac{1}{2}\left(m_{0}^{2}-\omega^{2}\right) \int_{M} u^{2} d v_{g}-\frac{1}{4} \int_{M}|u|^{4} d v_{g} .
$$

By (2.15), there exists $T_{0} \gg 1$ such that $I_{4}\left(T_{0} u_{\alpha}\right)<0$ for all $\alpha \gg 1$. There also holds that

$$
\begin{aligned}
\max _{0 \leq t \leq T_{0}} I_{4}\left(t u_{\alpha}\right) & \leq \max _{0 \leq t \leq T_{0}} \mathcal{F}_{4}\left(t u_{\alpha}\right)+C T_{0}^{2} \max _{0 \leq t \leq T_{0}} \int_{M} \Phi\left(t u_{\alpha}\right) u_{\alpha}^{2} d v_{g} \\
& \leq \frac{1}{4} J_{\lambda}\left(u_{\alpha}\right)^{2}+C T_{0}^{2} \max _{0 \leq t \leq T_{0}} \int_{M} \Phi\left(t u_{\alpha}\right) u_{\alpha}^{2} d v_{g}
\end{aligned}
$$

for all $\alpha$, where $\lambda=m_{0}^{2}-\omega^{2}$. By (2.14) and (2.17) we thus get that

$$
\max _{0 \leq t \leq T_{0}} I_{4}\left(t u_{\alpha}\right) \leq \frac{1}{K_{4}^{4}}\left(1+C\left(\frac{1}{6} S_{g}\left(x_{0}\right)-m_{0}^{2}+\omega^{2}\right) \varepsilon_{\alpha}^{2} \ln \varepsilon_{\alpha}+o\left(\varepsilon_{\alpha}^{2} \ln \varepsilon_{\alpha}\right)\right),
$$

where $C>0$ is independent of $\alpha$. By assumption the $\varepsilon_{\alpha}^{2} \ln \varepsilon_{\alpha}$ coefficient is positive. Let $u_{0}=u_{\alpha}$, where $\alpha \gg 1$ is sufficiently large such that

$$
\max _{0 \leq t \leq T_{0}} I_{4}\left(t u_{\alpha}\right) \leq \frac{1}{4 K_{4}^{4}}-\delta_{0}
$$

for some $\delta_{0}>0$. Since $u_{0}$ is now fixed, we can write that

$$
\max _{0 \leq t \leq T_{0}} I_{p}\left(t u_{0}\right) \leq\left(1+\delta_{\varepsilon}\right) \max _{0 \leq t \leq T_{0}} I_{4}\left(t u_{0}\right)
$$

for all $p \in(4-\varepsilon, 4)$, where $\delta_{\varepsilon}>0$ is such that $\delta_{\varepsilon} \rightarrow 0$ as $\varepsilon \rightarrow 0$. Noting that

$$
\begin{aligned}
I_{p}(u) & \geq \frac{1}{2} \int_{M}\left(|\nabla u|^{2}+\left(m_{0}^{2}-\omega^{2}\right) u^{2}\right) d v_{g}-\frac{1}{p} \int_{M}|u|^{p} d v_{g}, \\
& \geq C_{1}\|u\|_{H^{1}}^{2}-C_{2}\|u\|_{H^{1}}^{p}
\end{aligned}
$$

where $C_{1}, C_{2}>0$ are independent of $u$, there holds that there exist $\delta_{1}, \delta_{2}>0$ such that $\delta_{1}, \delta_{2}$ are as small as we want, and $I_{p}(u) \geq \delta_{2}$ for all $u$ such that $\|u\|_{H^{1}}=\delta_{1}$. 
As a conclusion, there exist $\delta_{0}>0$ and $\varepsilon>0$ such that (2.16) holds true for all $p \in(4-\varepsilon, 4)$. This ends the proof of the existence part in Theorem 0.3 .

There are always constant solutions to (0.3). By (2.16) the MPT solutions we obtain are distinct from these constant solutions in several situations, e.g. like on $S^{1}(T) \times S^{3}$ for $T \gg 1$ when $m_{1}^{2} / q \ll 1$.

\section{A PRIORI BOUNDS IN THE SUbCRITICAL CASE}

We prove the uniform bounds in the subcritical case of Theorem 0.2 . In what follows $p \in(2,4)$.

Proof of the uniform bounds in Theorem 0.2. Let $\left(\omega_{\alpha}\right)_{\alpha}$ be a sequence in $\left(-m_{0}, m_{0}\right)$ such that $\omega_{\alpha} \rightarrow \omega$ as $\alpha \rightarrow+\infty$ for some $\omega \in\left[-m_{0}, m_{0}\right]$. Also let $p \in(2,4)$ and $\left(\left(u_{\alpha}, v_{\alpha}\right)\right)_{\alpha}$ be a sequence of smooth positive solutions of (0.3) with phases $\omega_{\alpha}$. Then,

$$
\left\{\begin{array}{l}
\Delta_{g} u_{\alpha}+m_{0}^{2} u_{\alpha}=u_{\alpha}^{p-1}+\omega_{\alpha}^{2}\left(q v_{\alpha}-1\right)^{2} u_{\alpha} \\
\Delta_{g} v_{\alpha}+\left(m_{1}^{2}+q^{2} u_{\alpha}^{2}\right) v_{\alpha}=q u_{\alpha}^{2}
\end{array}\right.
$$

for all $\alpha$. By the second equation in (3.1), $0 \leq v_{\alpha} \leq \frac{1}{q}$ for all $\alpha$. Assume by contradiction that

$$
\max _{M} u_{\alpha} \rightarrow+\infty
$$

as $\alpha \rightarrow+\infty$. Let $x_{\alpha} \in M$ and $\mu_{\alpha}>0$ be given by

$$
u_{\alpha}\left(x_{\alpha}\right)=\max _{M} u_{\alpha}=\mu_{\alpha}^{-2 /(p-2)} .
$$

By (3.2), $\mu_{\alpha} \rightarrow 0$ as $\alpha \rightarrow+\infty$. Define $\tilde{u}_{\alpha}$ by

$$
\tilde{u}_{\alpha}(x)=\mu_{\alpha}^{\frac{2}{p-2}} u_{\alpha}\left(\exp _{x_{\alpha}}\left(\mu_{\alpha} x\right)\right)
$$

and $g_{\alpha}$ by $g_{\alpha}(x)=\left(\exp _{x_{\alpha}}^{\star} g\right)\left(\mu_{\alpha} x\right)$ for $x \in B_{0}\left(\delta \mu_{\alpha}^{-1}\right)$, where $\delta>0$ is small. Since $\mu_{\alpha} \rightarrow 0$, we get that $g_{\alpha} \rightarrow \xi$ in $C_{\text {loc }}^{2}\left(\mathbb{R}^{3}\right)$ as $\alpha \rightarrow+\infty$. Moreover, by (3.1),

$$
\Delta_{g_{\alpha}} \tilde{u}_{\alpha}+\mu_{\alpha}^{2} m_{0}^{2} \tilde{u}_{\alpha}=\tilde{u}_{\alpha}^{p-1}+\omega_{\alpha}^{2} \mu_{\alpha}^{2}\left(q \hat{v}_{\alpha}-1\right)^{2} \tilde{u}_{\alpha},
$$

where $\hat{v}_{\alpha}$ is given by $\hat{v}_{\alpha}(x)=v_{\alpha}\left(\exp _{x_{\alpha}}\left(\mu_{\alpha} x\right)\right)$. We have $\tilde{u}_{\alpha}(0)=1$ and $0 \leq \tilde{u}_{\alpha} \leq 1$. By (3.3) and standard elliptic theory arguments, we can write that, after passing to a subsequence, $\tilde{u}_{\alpha} \rightarrow u$ in $C_{l o c}^{1, \theta}\left(\mathbb{R}^{4}\right)$ as $\alpha \rightarrow+\infty$, where $u$ is such that $u(0)=1$ and $0 \leq u \leq 1$. Then

$$
\Delta u=u^{p-1}
$$

in $\mathbb{R}^{4}$, where $\Delta$ is the Euclidean Laplacian. It follows that $u$ is actually smooth and positive, and, since $2<p<4$, we get a contradiction with the Liouville result of Gidas and Spruck [24. As a conclusion, (3.2) is not possible and there exists $C>0$ such that

$$
u_{\alpha}+v_{\alpha} \leq C
$$

in $M$ for all $\alpha$. Coming back to (3.1) it follows that the sequences $\left(u_{\alpha}\right)_{\alpha}$ and $\left(v_{\alpha}\right)_{\alpha}$ are actually bounded in $H^{2, s}$ for all $s$. Pushing one step further the regularity argument they turn out to be bounded in $H^{3, s}$ for all $s$, and by the Sobolev embedding theorem we get that they are also bounded in $C^{2, \theta}, 0<\theta<1$. This ends the proof of the uniform bounds in Theorem 0.2 when $p \in(2,4)$. 
If we assume that $\omega_{\alpha} \rightarrow \omega$ as $\alpha \rightarrow+\infty$ for some $\omega \in\left(-m_{0}, m_{0}\right), p \in(2,4]$, and $u_{\alpha} \rightarrow u$ and $v_{\alpha} \rightarrow v$ in $C^{2}$ as $\alpha \rightarrow+\infty$, then $u>0, v>0$, and $u, v$ are smooth solutions of (0.3). Indeed, given $\varepsilon>0$ sufficiently small, since $m_{0}^{2}-\omega^{2}>0$, $\Delta_{g}+\left(m_{0}^{2}-\omega^{2}-\varepsilon\right)$ is coercive. There holds that $0 \leq v_{\alpha} \leq \frac{1}{q}$ for all $\alpha$. In particular, by (3.1) and the Sobolev inequality, for any $\alpha \gg 1$ sufficiently large,

$$
\begin{aligned}
& \int_{M}\left(\left|\nabla u_{\alpha}\right|^{2}+\left(m_{0}^{2}-\omega^{2}-\varepsilon\right) u_{\alpha}^{2}\right) d v_{g} \\
& \leq \int_{M}\left|\nabla u_{\alpha}\right|^{2} d v_{g}+m_{0}^{2} \int_{M} u_{\alpha}^{2} d v_{g}-\omega_{\alpha}^{2} \int_{M}\left(q v_{\alpha}^{2}-1\right)^{2} u_{\alpha}^{2} d v_{g} \\
& =\int_{M} u_{\alpha}^{p} d v_{g} \leq C\left(\int_{M}\left(\left|\nabla u_{\alpha}\right|^{2}+\left(m_{0}^{2}-\omega^{2}-\varepsilon\right) u_{\alpha}^{2}\right) d v_{g}\right)^{p / 2}
\end{aligned}
$$

for some $C>0$ independent of $\alpha$. This implies $u>0$ and then $v>0$. Obviously the positivity of $u$ and $v$ does not hold anymore if we allow $\omega^{2}=m_{0}^{2}$. Let $\left(\varepsilon_{\alpha}\right)_{\alpha}$ be a sequence of positive real numbers such that $\varepsilon_{\alpha} \rightarrow 0$ as $\alpha \rightarrow+\infty$. Let $u_{\alpha}=\varepsilon_{\alpha}$ and

$$
v_{\alpha}=\frac{q \varepsilon_{\alpha}^{2}}{m_{1}^{2}+q^{2} \varepsilon_{\alpha}^{2}} .
$$

Then $u_{\alpha} \rightarrow 0$ and $v_{\alpha} \rightarrow 0$ in $C^{2}$ as $\alpha \rightarrow+\infty$, and we do have that $\left(u_{\alpha}, v_{\alpha}\right)$ solves (3.1), where

$$
\omega_{\alpha}^{2}=\frac{1}{\left(q v_{\alpha}-1\right)^{2}}\left(m_{0}^{2}-\varepsilon_{\alpha}^{p-2}\right) .
$$

In this case $\omega_{\alpha}^{2} \rightarrow m_{0}^{2}$ as $\alpha \rightarrow+\infty$ and the construction provides a counter example to the above statement about the positivity of $u$ and $v$.

\section{A PRIORI BOUNDS IN THE CRITICAL CASE}

In what follows we let $(M, g)$ be a smooth compact 4-dimensional Riemannian manifold, $m_{0}, m_{1}>0$, and $\left(\omega_{\alpha}\right)_{\alpha}$ be a sequence in $\left(-m_{0}, m_{0}\right)$ such that $\omega_{\alpha} \rightarrow \omega$ as $\alpha \rightarrow+\infty$ for some $\omega \in\left[-m_{0}, m_{0}\right]$. Also we let $\left(\left(u_{\alpha}, v_{\alpha}\right)\right)_{\alpha}$ be a sequence of smooth positive solutions of (0.3) with phases $\omega_{\alpha}$ and $p=4$. Namely,

$$
\left\{\begin{array}{l}
\Delta_{g} u_{\alpha}+m_{0}^{2} u_{\alpha}=u_{\alpha}^{3}+\omega_{\alpha}^{2}\left(q v_{\alpha}-1\right)^{2} u_{\alpha} \\
\Delta_{g} v_{\alpha}+\left(m_{1}^{2}+q^{2} u_{\alpha}^{2}\right) v_{\alpha}=q u_{\alpha}^{2}
\end{array}\right.
$$

for all $\alpha$. By the second equation in (4.1), $0 \leq v_{\alpha} \leq \frac{1}{q}$ for all $\alpha$. In particular, if we let

$$
h_{\alpha}=m_{0}^{2}-\omega_{\alpha}^{2}\left(q v_{\alpha}-1\right)^{2},
$$

then $\left\|h_{\alpha}\right\|_{L^{\infty}} \leq C$ for all $\alpha$, where $C>0$ is independent of $\alpha$. Assume by contradiction that

$$
\max _{M} u_{\alpha} \rightarrow+\infty
$$

as $\alpha \rightarrow+\infty$. In what follows we let $\left(x_{\alpha}\right)_{\alpha}$ be a sequence of points in $M$, and $\left(\rho_{\alpha}\right)_{\alpha}$ be a sequence of positive real numbers, $0<\rho_{\alpha}<i_{g} / 7$ for all $\alpha$, where $i_{g}$ is the injectivity radius of $(M, g)$. We assume that the $x_{\alpha}$ 's and $\rho_{\alpha}$ 's satisfy

$$
\left\{\begin{array}{l}
\nabla u_{\alpha}\left(x_{\alpha}\right)=0 \text { for all } \alpha \\
d_{g}\left(x_{\alpha}, x\right) u_{\alpha}(x) \leq C \text { for all } x \in B_{x_{\alpha}}\left(7 \rho_{\alpha}\right) \text { and all } \alpha, \\
\lim _{\alpha \rightarrow+\infty} \rho_{\alpha} \sup _{B_{x_{\alpha}}\left(6 \rho_{\alpha}\right)} u_{\alpha}(x)=+\infty
\end{array}\right.
$$


We let $\mu_{\alpha}$ be given by

$$
\mu_{\alpha}=u_{\alpha}\left(x_{\alpha}\right)^{-1} \text {. }
$$

Since the $h_{\alpha}$ 's in (4.2) are $L^{\infty}$-bounded we can apply the asymptotic analysis in Druet and Hebey [18] and Druet, Hebey and Vétois [22. In particular, we get that $\frac{\rho_{\alpha}}{\mu_{\alpha}} \rightarrow+\infty$ as $\alpha \rightarrow+\infty$ and that

$$
\mu_{\alpha} u_{\alpha}\left(\exp _{x_{\alpha}}\left(\mu_{\alpha} x\right)\right) \rightarrow\left(1+\frac{|x|^{2}}{8}\right)^{-1}
$$

in $C_{l o c}^{1}\left(\mathbb{R}^{4}\right)$ as $\alpha \rightarrow+\infty$, where $\mu_{\alpha}$ is as in (4.5). As a consequence, $\mu_{\alpha} \rightarrow 0$ as $\alpha \rightarrow+\infty$. Now we define $\varphi_{\alpha}:\left(0, \rho_{\alpha}\right) \mapsto \mathbb{R}^{+}$by

$$
\varphi_{\alpha}(r)=\frac{1}{\left|\partial B_{x_{\alpha}}(r)\right|_{g}} \int_{\partial B_{x_{\alpha}}(r)} u_{\alpha} d \sigma_{g},
$$

where $\left|\partial B_{x_{\alpha}}(r)\right|_{g}$ is the volume of the sphere of center $x_{\alpha}$ and radius $r$ for the induced metric. Let $\Lambda=4 \sqrt{2}$. We define $r_{\alpha} \in\left[\Lambda \mu_{\alpha}, \rho_{\alpha}\right]$ by

$$
r_{\alpha}=\sup \left\{r \in\left[\Lambda \mu_{\alpha}, \rho_{\alpha}\right] \text { s.t. }\left(s \varphi_{\alpha}(s)\right)^{\prime} \leq 0 \text { in }\left[\Lambda \mu_{\alpha}, r\right]\right\} .
$$

It follows from (4.6) that

$$
\frac{r_{\alpha}}{\mu_{\alpha}} \rightarrow+\infty
$$

as $\alpha \rightarrow+\infty$, while the definition of $r_{\alpha}$ gives that

$$
r \varphi_{\alpha}(r) \text { is non-increasing in }\left[\Lambda \mu_{\alpha}, r_{\alpha}\right]
$$

and that

$$
\left(r \varphi_{\alpha}(r)\right)^{\prime}\left(r_{\alpha}\right)=0 \text { if } r_{\alpha}<\rho_{\alpha} .
$$

Let $B_{\alpha}$ be defined in $M$ by

$$
B_{\alpha}(x)=\frac{\mu_{\alpha}}{\mu_{\alpha}^{2}+\frac{d_{g}\left(x_{\alpha}, x\right)^{2}}{8}},
$$

where $\mu_{\alpha}$ is as in (4.5). The following sharp estimates, see Druet, Hebey and Robert 21] and Druet, Hebey and Vétois [22], hold true.

Lemma 4.1. Let $(M, g)$ be a smooth compact Riemannian 4-dimensional manifold, and $\left(\left(u_{\alpha}, v_{\alpha}\right)\right)_{\alpha}$ be a sequence of smooth positive solutions of (4.1) such that (4.3) holds true. Let $\left(x_{\alpha}\right)_{\alpha}$ and $\left(\rho_{\alpha}\right)_{\alpha}$ be such that (4.4) hold true, and let $R \geq 6$ be such that $R r_{\alpha} \leq 6 \rho_{\alpha}$ for all $\alpha \gg 1$. There exists $C>0$ such that, after passing to a subsequence,

$$
u_{\alpha}(x)+d_{g}\left(x_{\alpha}, x\right)\left|\nabla u_{\alpha}(x)\right| \leq C \mu_{\alpha} d_{g}\left(x_{\alpha}, x\right)^{-2}
$$

for all $x \in B_{x_{\alpha}}\left(\frac{R}{2} r_{\alpha}\right) \backslash\left\{x_{\alpha}\right\}$ and all $\alpha$, where $\mu_{\alpha}$ is as in (4.5), and where $r_{\alpha}$ is as in (4.8). In addition, there also exist $C>0$ and $\left(\varepsilon_{\alpha}\right)_{\alpha}$ such that

$$
\left|u_{\alpha}-B_{\alpha}\right| \leq C \mu_{\alpha}\left(r_{\alpha}^{-2}+S_{\alpha}\right)+\varepsilon_{\alpha} B_{\alpha}
$$

in $B_{x_{\alpha}}\left(2 r_{\alpha}\right) \backslash\left\{x_{\alpha}\right\}$ for all $\alpha$, where $\varepsilon_{\alpha} \rightarrow 0$ as $\alpha \rightarrow+\infty$ and $S_{\alpha}(x)=d_{g}\left(x_{\alpha}, x\right)^{-1}$ for $x \in M \backslash\left\{x_{\alpha}\right\}$.

Lemma 4.1 provide a sharp control on the $u_{\alpha}$ 's, but we need more to conclude. We prove that the following fundamental asymptotic estimate holds true. Lemma 4.2 is the key estimate we need to prove the a priori bounds in the critical case discussed in this section. 
Lemma 4.2. Let $(M, g)$ be a smooth compact Riemannian 4-dimensional manifold and $\left(\left(u_{\alpha}, v_{\alpha}\right)\right)_{\alpha}$ be a sequence of smooth positive solutions of (4.1) such that (4.3) holds true. Let $\left(x_{\alpha}\right)_{\alpha}$ and $\left(\rho_{\alpha}\right)_{\alpha}$ be such that (4.4) holds true. Assume (0.7). There holds that $r_{\alpha} \rightarrow 0$ as $\alpha \rightarrow+\infty$, where $r_{\alpha}$ is as in (4.8). Moreover $\rho_{\alpha}=O\left(r_{\alpha}\right)$ and

$$
r_{\alpha}^{2} \mu_{\alpha}^{-1} u_{\alpha}\left(\exp _{x_{\alpha}}\left(r_{\alpha} x\right)\right) \rightarrow \frac{8}{|x|^{2}}+\mathcal{H}(x)
$$

in $C_{\text {loc }}^{2}\left(B_{0}(2) \backslash\{0\}\right)$ as $\alpha \rightarrow+\infty$, where $\mu_{\alpha}$ is as in (4.5), and $\mathcal{H}$ is a harmonic function in $B_{0}(2)$ which satisfies that $\mathcal{H}(0) \leq 0$.

Proof of Lemma 4.2. Let $R \geq 6$ be such that $R r_{\alpha} \leq 6 \rho_{\alpha}$ for $\alpha \gg 1$. We assume first that $r_{\alpha} \rightarrow 0$ as $\alpha \rightarrow+\infty$. For $x \in B_{0}(3)$ we define

$$
\begin{aligned}
& \tilde{u}_{\alpha}(x)=r_{\alpha}^{2} \mu_{\alpha}^{-1} u_{\alpha}\left(\exp _{x_{\alpha}}\left(r_{\alpha} x\right)\right), \\
& \tilde{g}_{\alpha}(x)=\left(\exp _{x_{\alpha}}^{\star} g\right)\left(r_{\alpha} x\right), \text { and } \\
& \tilde{h}_{\alpha}(x)=h_{\alpha}\left(\exp _{x_{\alpha}}\left(r_{\alpha} x\right)\right),
\end{aligned}
$$

where $h_{\alpha}$ is as in (4.2). Since $r_{\alpha} \rightarrow 0$ as $\alpha \rightarrow+\infty$, we have that $\tilde{g}_{\alpha} \rightarrow \xi$ in $C_{\text {loc }}^{2}\left(\mathbb{R}^{n}\right)$ as $\alpha \rightarrow+\infty$, where $\xi$ is the Euclidean metric. Thanks to Lemma 4.1 .

$$
\left|\tilde{u}_{\alpha}(x)\right| \leq C|x|^{-2}
$$

in $B_{0}\left(\frac{R}{2}\right) \backslash\{0\}$. By (4.1),

$$
\Delta_{\tilde{g}_{\alpha}} \tilde{u}_{\alpha}+r_{\alpha}^{2} \tilde{h}_{\alpha} \tilde{u}_{\alpha}=\left(\frac{\mu_{\alpha}}{r_{\alpha}}\right)^{2} \tilde{u}_{\alpha}^{3}
$$

in $B_{0}\left(\frac{R}{2}\right)$. Thanks to (4.9) and by standard elliptic theory, we then deduce that, after passing to a subsequence,

$$
\tilde{u}_{\alpha} \rightarrow \tilde{u}
$$

in $C_{l o c}^{2}\left(B_{0}\left(\frac{R}{2}\right) \backslash\{0\}\right)$ as $\alpha \rightarrow+\infty$, where $\mathcal{W}$ satisfies $\Delta \tilde{u}=0$ in $B_{0}\left(\frac{R}{2}\right) \backslash\{0\}$ and $\Delta$ is the Euclidean Laplace Beltrami operator. Moreover, thanks to (4.16), we know that

$$
|\tilde{u}(x)| \leq C|x|^{-2}
$$

in $B_{0}\left(\frac{R}{2}\right) \backslash\{0\}$. Thus we can write that

$$
\tilde{u}(x)=\frac{\Lambda}{|x|^{2}}+\mathcal{H}(x)
$$

where $\Lambda \geq 0$ and $\mathcal{H}$ satisfies $\Delta \mathcal{H}=0$ in $B_{0}\left(\frac{R}{2}\right)$. In order to see that $\Lambda=8$, it is sufficient to integrate (4.17) in $B_{0}(1)$ to get that

$$
-\int_{\partial B_{0}(1)} \partial_{\nu} \tilde{u}_{\alpha} d \sigma_{\tilde{g}_{\alpha}}=\left(\frac{\mu_{\alpha}}{r_{\alpha}}\right)^{2} \int_{B_{0}(1)} \tilde{u}_{\alpha}^{3} d v_{\tilde{g}_{\alpha}}-r_{\alpha}^{2} \int_{B_{0}(1)} \tilde{h}_{\alpha} \tilde{u}_{\alpha} d v_{\tilde{g}_{\alpha}} .
$$

By (4.16),

$$
\int_{B_{0}(1)} \tilde{u}_{\alpha} d v_{\tilde{g}_{\alpha}} \leq C
$$

and by changing $x$ into $\frac{\mu_{\alpha}}{r_{\alpha}} x$, we can write that

$$
\int_{B_{0}(1)} \tilde{u}_{\alpha}^{3} d v_{g_{\alpha}}=r_{\alpha}^{2} \mu_{\alpha}^{-2} \int_{B_{0}\left(\frac{r_{\alpha}}{\mu_{\alpha}}\right)} \hat{u}_{\alpha}^{3} d v_{\hat{g}_{\alpha}},
$$


where $\hat{u}_{\alpha}(x)=\mu_{\alpha} u_{\alpha}\left(\exp _{x_{\alpha}}\left(\mu_{\alpha} x\right)\right)$ and $\hat{g}_{\alpha}(x)=\left(\exp _{x_{\alpha}}^{\star} g\right)\left(\mu_{\alpha} x\right)$. By (4.6) and Lemma 4.1, we then get that

$$
\lim _{\alpha \rightarrow+\infty}\left(\frac{\mu_{\alpha}}{r_{\alpha}}\right)^{2} \int_{B_{0}(1)} \tilde{u}_{\alpha}^{3} d v_{\tilde{g}_{\alpha}}=16 \omega_{3} .
$$

Noting that by (4.18) and (4.20),

$$
\lim _{\alpha \rightarrow+\infty} \int_{\partial B_{0}(1)} \partial_{\nu} \tilde{u}_{\alpha} d \sigma_{\tilde{g}_{\alpha}}=-2 \omega_{3} \Lambda,
$$

we get that $\Lambda=8$ thanks to (4.22) (4.24) by passing into the limit in (4.21) as $\alpha \rightarrow+\infty$. At this point we claim that there exists $\beta \in(0,1]$ and $C>0$ such that

$$
v_{\alpha} \leq C u_{\alpha}^{\beta} \text { in } M
$$

for all $\alpha$. Let $x_{\alpha} \in M$ be a point where $\frac{v_{\alpha}}{u_{\alpha}^{\beta}}$ is maximum. Then,

$$
\frac{\Delta_{g} v_{\alpha}\left(x_{\alpha}\right)}{v_{\alpha}\left(x_{\alpha}\right)} \geq \frac{\Delta_{g} u_{\alpha}^{\beta}\left(x_{\alpha}\right)}{u_{\alpha}^{\beta}\left(x_{\alpha}\right)}
$$

and it follows from (4.1) that

$$
\begin{aligned}
& q \frac{u_{\alpha}\left(x_{\alpha}\right)^{2}}{v_{\alpha}\left(x_{\alpha}\right)}-m_{1}^{2}-q^{2} u_{\alpha}\left(x_{\alpha}\right)^{2} \\
& \geq-\beta(\beta-1) \frac{\left|\nabla u_{\alpha}\left(x_{\alpha}\right)\right|^{2}}{u_{\alpha}\left(x_{\alpha}\right)^{2}}+\beta u_{\alpha}\left(x_{\alpha}\right)^{2}-\beta m_{0}^{2}+\beta \omega_{\alpha}^{2}\left(q v_{\alpha}\left(x_{\alpha}\right)-1\right)^{2} .
\end{aligned}
$$

Choosing $\beta \in(0,1]$ such that $m_{1}^{2}-\beta m_{0}^{2}>0$, since $0<v_{\alpha} \leq \frac{1}{q}$, we get that $u_{\alpha}^{\beta}\left(x_{\alpha}\right) \geq C v_{\alpha}\left(x_{\alpha}\right)$ for some $C>0$ independent of $\alpha$. This proves 4.25). In what follows we let $X_{\alpha}$ be the 1 -form given by

$$
X_{\alpha}(x)=\left(1-\frac{1}{18} \operatorname{Rc}_{g}^{\sharp}(x) \cdot\left(\nabla f_{\alpha}(x), \nabla f_{\alpha}(x)\right)\right) \nabla f_{\alpha}(x),
$$

where $f_{\alpha}(x)=\frac{1}{2} d_{g}\left(x_{\alpha}, x\right)^{2}, \mathrm{Rc}_{g}$ is the Ricci curvature of $g$, and $\sharp$ is the musical isomorphism. We apply the Pohozaev identity in Druet-Hebey 19 with the vector field $X_{\alpha}$ to $u_{\alpha}$ in $B_{x_{\alpha}}\left(r_{\alpha}\right)$. We separate the regular part $A_{\alpha}=m_{0}^{2}-\omega_{\alpha}^{2}$ from the singular part in $h_{\alpha}$. Then, $h_{\alpha}=A_{\alpha}+O\left(v_{\alpha}\right)$ and we get that

$$
\begin{aligned}
& \int_{B_{x_{\alpha}}\left(r_{\alpha}\right)} A_{\alpha} u_{\alpha} X_{\alpha}\left(\nabla u_{\alpha}\right) d v_{g}+\frac{1}{8} \int_{B_{x_{\alpha}}\left(r_{\alpha}\right)}\left(\Delta_{g} \operatorname{div}_{g} X_{\alpha}\right) u_{\alpha}^{2} d v_{g} \\
& +\frac{1}{4} \int_{B_{x_{\alpha}}\left(r_{\alpha}\right)}\left(\operatorname{div}_{g} X_{\alpha}\right) A_{\alpha} u_{\alpha}^{2} d v_{g} \\
& =Q_{1, \alpha}+Q_{2, \alpha}+Q_{3, \alpha}+O\left(\int_{B_{x_{\alpha}}\left(r_{\alpha}\right)} v_{\alpha} u_{\alpha}^{2} d v_{g}\right) \\
& +O\left(\int_{B_{x_{\alpha}}\left(r_{\alpha}\right)} v_{\alpha} u_{\alpha}\left|X_{\alpha}\left(\nabla u_{\alpha}\right)\right| d v_{g}\right),
\end{aligned}
$$


where

$$
\begin{aligned}
& Q_{1, \alpha}= \frac{1}{4} \int_{\partial B_{x_{\alpha}}\left(r_{\alpha}\right)}\left(\operatorname{div}_{g} X_{\alpha}\right) u_{\alpha} \partial_{\nu} u_{\alpha} d \sigma_{g} \\
&-\int_{\partial B_{x_{\alpha}}\left(r_{\alpha}\right)}\left(\frac{1}{2} X_{\alpha}(\nu)\left|\nabla u_{\alpha}\right|^{2}-X_{\alpha}\left(\nabla u_{\alpha}\right) \partial_{\nu} u_{\alpha}\right) d \sigma_{g}, \\
& Q_{2, \alpha}=-\sum_{i=1}^{p} \int_{B_{x_{\alpha}}\left(r_{\alpha}\right)}\left(\nabla X_{\alpha}-\frac{1}{4}\left(\operatorname{div}_{g} X_{\alpha}\right) g\right)^{\sharp}\left(\nabla u_{\alpha}, \nabla u_{\alpha}\right) d v_{g}, \\
& Q_{3, \alpha}=\frac{1}{4} \int_{\partial B_{x_{\alpha}}\left(r_{\alpha}\right)} X_{\alpha}(\nu) u_{\alpha}^{4} d \sigma_{g}-\frac{1}{8} \int_{\partial B_{x_{\alpha}}\left(r_{\alpha}\right)}\left(\partial_{\nu} \operatorname{div}_{g} X_{\alpha}\right) u_{\alpha}^{2} d \sigma_{g},
\end{aligned}
$$

and $\nu$ is the unit outward normal derivative to $B_{x_{\alpha}}\left(r_{\alpha}\right)$. We have that

$$
\begin{aligned}
& \left.\left|X_{\alpha}(x)\right|=O\left(d_{g}\left(x_{\alpha}, x\right)\right), \operatorname{div}_{g} X_{\alpha}(x)=n+O\left(d_{g}\left(x_{\alpha}, x\right)^{2}\right)\right), \\
& \left|\nabla\left(\operatorname{div}_{g} X_{\alpha}\right)(x)\right|=O\left(d_{g}\left(x_{\alpha}, x\right)\right), \\
& \text { and } \Delta_{g}\left(\operatorname{div}_{g} X_{\alpha}\right)(x)=\frac{4}{3} S_{g}\left(x_{\alpha}\right)+O\left(d_{g}\left(x_{\alpha}, x\right)\right) .
\end{aligned}
$$

Following Druet, Hebey and Vétois [22] we get from Lemma 4.1, (4.28) and (4.29) that

$$
\begin{aligned}
Q_{1, \alpha} & =-64 \omega_{3}\left(m_{0}^{2}-\omega^{2}-\frac{1}{6} S_{g}\left(x_{0}\right)\right) \mu_{\alpha}^{2} \ln \frac{r_{\alpha}}{\mu_{\alpha}} \\
& +o\left(\mu_{\alpha}^{2} \ln \frac{1}{\mu_{\alpha}}\right)+o\left(\mu_{\alpha}^{2} r_{\alpha}^{-2}\right)+O\left(\int_{B_{x_{\alpha}}\left(r_{\alpha}\right)} v_{\alpha} u_{\alpha}^{2} d v_{g}\right) \\
& +O\left(\int_{B_{x_{\alpha}}\left(r_{\alpha}\right)} v_{\alpha} u_{\alpha}\left|X_{\alpha}\left(\nabla u_{\alpha}\right)\right| d v_{g}\right)
\end{aligned}
$$

where $x_{\alpha} \rightarrow x_{0}$ as $\alpha \rightarrow+\infty$. By Lemma 4.1 and (4.29) there also holds that

$$
Q_{1, \alpha}=O\left(\mu_{\alpha}^{2} r_{\alpha}^{-2}\right)
$$

At this point we decompose $v_{\alpha}$ into a quasi-harmonic part with nonzero Dirichlet boundary condition and a quasi-Poisson part with zero Dirichler boundary condition. More precisely, we write that

$$
v_{\alpha}=w_{1, \alpha}+w_{2, \alpha}
$$

in $B_{\alpha}=B_{x_{\alpha}}\left(\hat{r}_{\alpha}\right)$, where $\hat{r}_{\alpha}=\frac{5}{2} r_{\alpha}$, and $w_{1, \alpha}, w_{2, \alpha}$ are given by

$$
\left\{\begin{array}{l}
\Delta_{g} w_{1, \alpha}+m_{1}^{2} w_{1, \alpha}=0 \text { in } B_{\alpha} \\
w_{1, \alpha}=v_{\alpha} \text { on } \partial B_{\alpha},
\end{array}\right.
$$

and if $W_{\alpha}=\Delta_{g} v_{\alpha}+m_{1}^{2} v_{\alpha}$, by

$$
\left\{\begin{array}{l}
\Delta_{g} w_{2, \alpha}+m_{1}^{2} w_{2, \alpha}=W_{\alpha} \text { in } B_{\alpha} \\
w_{2, \alpha}=0 \text { on } \partial B_{\alpha} .
\end{array}\right.
$$

Let $G_{\alpha}$ be the Green's function of $\Delta_{g}+m_{1}^{2}$ in $B_{\alpha}$ with zero Dirichlet boundary condition on $\partial B_{\alpha}$. By the maximum principle, considering the Green's function on a larger ball of radius $i_{g}$, we obtain by comparison of the two Green's functions that 
there exists $C>0$ such that $G_{\alpha}(x, y) \leq C d_{g}(x, y)^{-2}$ for all $x \neq y$ in $B_{\alpha}$. Writing that

it follows that

$$
w_{2, \alpha}(x)=\int_{B_{\alpha}} G_{\alpha}(x, y) W_{\alpha}(y) d v_{g}(y)
$$

$$
\left|w_{2, \alpha}(x)\right| \leq C \int_{B_{\alpha}} \frac{u_{\alpha}^{2}(y) d v_{g}(y)}{d_{g}(x, y)^{2}}
$$

By (4.6) and Lemma 4.1 we can write that

$$
u_{\alpha}(x) \leq \frac{C \mu_{\alpha}}{\mu_{\alpha}^{2}+d_{g}\left(x_{\alpha}, x\right)^{2}}
$$

in $B_{\alpha}$. Combining (4.35) and (4.36) we then get that

$$
\left|w_{2, \alpha}(x)\right| \leq C \frac{\mu_{\alpha}^{2} \ln \left(2+\frac{d_{g}\left(x_{\alpha}, x\right)^{2}}{\mu_{\alpha}^{2}}\right)}{\mu_{\alpha}^{2}+d_{g}\left(x_{\alpha}, x\right)^{2}} .
$$

Independently, by the maximum principle, the $w_{1, \alpha}$ 's satisfy that $0 \leq w_{1, \alpha} \leq \frac{1}{q}$. Let $\hat{g}_{\alpha}(x)=\left(\exp _{x_{\alpha}}^{\star} g\right)\left(\hat{r}_{\alpha} x\right)$ and $\hat{w}_{1, \alpha}(x)=w_{1, \alpha}\left(\exp _{x_{\alpha}}\left(\hat{r}_{\alpha} x\right)\right)$. There holds

$$
\left\{\begin{array}{l}
\Delta_{\hat{g}_{\alpha}} \hat{w}_{1, \alpha}+m_{1}^{2} \hat{r}_{\alpha}^{2} \hat{w}_{1, \alpha}=0 \text { in } B \\
w_{1, \alpha}=\hat{v}_{\alpha} \text { on } \partial B
\end{array}\right.
$$

where $B=B_{0}(1) \subset \mathbb{R}^{4}$, and $\hat{v}_{\alpha}(x)=v_{\alpha}\left(\exp _{x_{\alpha}}\left(\hat{r}_{\alpha} x\right)\right)$. At this point we claim that

$$
r_{\alpha} \rightarrow 0
$$

as $\alpha \rightarrow+\infty$. In order to prove (4.39) we proceed by contradiction and assume that $r_{\alpha} \geq \delta_{0}>0$ for all $\alpha \gg 1$. By Lemma 4.1 and (4.25),

$$
v_{\alpha} \leq C \mu_{\alpha}^{\beta} \text { in } M \backslash B_{x_{\alpha}}\left(r_{\alpha}\right),
$$

where $C>0$ is independent of $\alpha$ since we assumed $r_{\alpha} \geq \delta_{0}>0$. In particular, $\left\|v_{\alpha}\right\|_{L^{\infty}\left(\partial B_{\alpha}\right)} \rightarrow 0$ as $\alpha \rightarrow+\infty$. Then $\left\|\hat{v}_{\alpha}\right\|_{L^{\infty}(\partial B)} \rightarrow 0$ as $\alpha \rightarrow+\infty$, and it follows from the maximum principle and (4.38) that $\left\|\hat{w}_{1, \alpha}\right\|_{L^{\infty}(B)} \rightarrow 0$ as $\alpha \rightarrow+\infty$. In particular, $\left\|w_{1, \alpha}\right\|_{L^{\infty}\left(B_{\alpha}\right)} \rightarrow 0$ as $\alpha \rightarrow+\infty$. By (4.32) and (4.37), thanks to what we just obtained about the $w_{1, \alpha}$ 's, we get that $\left\|v_{\alpha}\right\|_{L^{\infty}\left(B_{\alpha}\right)} \rightarrow 0$ as $\alpha \rightarrow+\infty$. Then, by Lemma 4.1 and (4.29) we get that

$$
\begin{aligned}
& \int_{B_{x_{\alpha}}\left(r_{\alpha}\right)} v_{\alpha} u_{\alpha}^{2} d v_{g}=o\left(\mu_{\alpha}^{2} \ln \frac{1}{\mu_{\alpha}}\right) \\
& \int_{B_{x_{\alpha}}\left(r_{\alpha}\right)} u_{\alpha} v_{\alpha}\left|X_{\alpha}\left(\nabla u_{\alpha}\right)\right| d v_{g}=o\left(\mu_{\alpha}^{2} \ln \frac{1}{\mu_{\alpha}}\right) .
\end{aligned}
$$

and by (4.30) and (4.31), we obtain a contradiction with (0.7). This proves (4.39). By (4.18), (4.19) and (4.20) we get with (4.39) that

$$
Q_{1, \alpha}=-\left(128 \omega_{3} \mathcal{H}(0)+o(1)\right) \mu_{\alpha}^{2} r_{\alpha}^{-2} .
$$

Now we distinguish the two cases:

(i) $r_{\alpha}^{2} \ln \frac{r_{\alpha}}{\mu_{\alpha}} \rightarrow 0$ as $\alpha \rightarrow+\infty$, and

(ii) $r_{\alpha}^{2} \ln \frac{r_{\alpha}}{\mu_{\alpha}} \geq \delta_{0}>0$ for all $\alpha$. 
In case (i), since $v_{\alpha}=O(1)$, we get from Lemma 4.1 and (4.29) that

$$
\begin{aligned}
& \int_{B_{x_{\alpha}}\left(r_{\alpha}\right)} v_{\alpha} u_{\alpha}^{2} d v_{g}=O\left(\mu_{\alpha}^{2} \ln \frac{r_{\alpha}}{\mu_{\alpha}}\right), \\
& \int_{B_{x_{\alpha}}\left(r_{\alpha}\right)} v_{\alpha} u_{\alpha}\left|X_{\alpha}\left(\nabla u_{\alpha}\right)\right| d v_{g}=O\left(\mu_{\alpha}^{2} \ln \frac{r_{\alpha}}{\mu_{\alpha}}\right) .
\end{aligned}
$$

Since there also holds that $r_{\alpha}^{2} \ln \frac{1}{\mu_{\alpha}} \rightarrow+\infty$ it follows from (4.30), (4.42) and (4.43) that $\mathcal{H}(0)=0$. Now we assume (ii). From (ii) we get that $r_{\alpha} \geq C\left(\ln \frac{1}{\mu_{\alpha}}\right)^{-1 / 2}$ and by (4.25) we obtain that

$$
v_{\alpha} \leq C\left(\mu_{\alpha} \ln \frac{1}{\mu_{\alpha}}\right)^{\beta} \text { in } M \backslash B_{x_{\alpha}}\left(r_{\alpha}\right) .
$$

In particular, $\left\|v_{\alpha}\right\|_{L^{\infty}\left(\partial B_{\alpha}\right)} \rightarrow 0$ as $\alpha \rightarrow+\infty$. Then $\left\|\hat{v}_{\alpha}\right\|_{L^{\infty}(\partial B)} \rightarrow 0$ as $\alpha \rightarrow+\infty$, and it follows from the maximum principle and (4.38) that $\left\|\hat{w}_{1, \alpha}\right\|_{L^{\infty}(B)} \rightarrow 0$ as $\alpha \rightarrow+\infty$. In particular, $\left\|w_{1, \alpha}\right\|_{L^{\infty}\left(B_{\alpha}\right)} \rightarrow 0$ as $\alpha \rightarrow+\infty$ and we get with (4.32), Lemma 4.1, and (4.29), that

$$
\begin{aligned}
& \int_{B_{x_{\alpha}}\left(r_{\alpha}\right)} v_{\alpha} u_{\alpha}^{2} d v_{g}=\int_{B_{x_{\alpha}}\left(r_{\alpha}\right)} w_{2, \alpha} u_{\alpha}^{2} d v_{g}+o\left(\mu_{\alpha}^{2} \ln \frac{r_{\alpha}}{\mu_{\alpha}}\right), \\
& \int_{B_{x_{\alpha}}\left(r_{\alpha}\right)} v_{\alpha} u_{\alpha}\left|X_{\alpha}\left(\nabla u_{\alpha}\right)\right| d v_{g}= \\
& \int_{B_{x_{\alpha}}\left(r_{\alpha}\right)} w_{2, \alpha} u_{\alpha}\left|X_{\alpha}\left(\nabla u_{\alpha}\right)\right| d v_{g}+o\left(\mu_{\alpha}^{2} \ln \frac{r_{\alpha}}{\mu_{\alpha}}\right) .
\end{aligned}
$$

There holds,

$$
\Delta_{g} w_{2, \alpha}+m_{1}^{2} w_{2, \alpha}=q\left(1-q v_{\alpha}\right) u_{\alpha}^{2} .
$$

Let $\eta: \mathbb{R}^{n} \rightarrow \mathbb{R}$ be such that $\eta$ is smooth, $0 \leq \eta \leq 1, \eta=1$ in $B_{0}(1)$, and $\eta=0$ in $\mathbb{R}^{n} \backslash B_{0}(2)$. We define

$$
\eta_{\alpha}(x)=\eta\left(\frac{d_{g}\left(x_{\alpha}, x\right)}{r_{\alpha}}\right)
$$

so that $\eta_{\alpha}=1$ in $B_{x_{\alpha}}\left(r_{\alpha}\right)$ and $\eta_{\alpha}=0$ in $M \backslash B_{x_{\alpha}}\left(2 r_{\alpha}\right)$. By Hölder's inequalities,

$$
\begin{aligned}
& \int_{B_{x_{\alpha}}\left(r_{\alpha}\right)} w_{2, \alpha} u_{\alpha}^{2} d v_{g} \leq\left(\int_{B_{x_{\alpha}}\left(r_{\alpha}\right)} w_{2, \alpha}^{4} d v_{g}\right)^{1 / 4}\left(\int_{B_{x_{\alpha}}\left(r_{\alpha}\right)} u_{\alpha}^{8 / 3} d v_{g}\right)^{3 / 4} \text { and } \\
& \int_{B_{x_{\alpha}}\left(r_{\alpha}\right)} u_{\alpha} w_{2, \alpha}\left|X_{\alpha}\left(\nabla u_{\alpha}\right)\right| d v_{g} \\
& \leq\left(\int_{B_{x_{\alpha}}\left(r_{\alpha}\right)} w_{2, \alpha}^{4} d v_{g}\right)^{1 / 4}\left(\int_{B_{x_{\alpha}}\left(r_{\alpha}\right)}\left|u_{\alpha} X_{\alpha}\left(\nabla u_{\alpha}\right)\right|^{4 / 3} d v_{g}\right)^{3 / 4},
\end{aligned}
$$

while by Lemma 4.1 and (4.29) there holds that

$$
\begin{aligned}
& \int_{B_{x_{\alpha}}\left(r_{\alpha}\right)} u_{\alpha}^{8 / 3} d v_{g}=O\left(\mu_{\alpha}^{4 / 3}\right) \text { and } \\
& \int_{B_{x_{\alpha}}\left(r_{\alpha}\right)}\left|u_{\alpha} X_{\alpha}\left(\nabla u_{\alpha}\right)\right|^{4 / 3} d v_{g}=O\left(\mu_{\alpha}^{4 / 3}\right) .
\end{aligned}
$$


Multiplying (4.45) by $\eta_{\alpha}^{2} w_{2, \alpha}$, and integrating over $M$, we get that

$$
\int_{M}\left(\Delta_{g} w_{2, \alpha}+m_{1}^{2} w_{2, \alpha}\right) \eta_{\alpha}^{2} w_{2, \alpha} d v_{g} \leq q \int_{M} u_{\alpha}^{2} w_{2, \alpha} \eta_{\alpha}^{2} d v_{g} .
$$

By Hölder's and Sobolev inequalities, and by (4.48),

$$
\int_{M} u_{\alpha}^{2} w_{2, \alpha} \eta_{\alpha}^{2} d v_{g} \leq C \mu_{\alpha}\left\|\eta_{\alpha} w_{2, \alpha}\right\|_{H^{1}}
$$

and it follows from (4.49) and (4.50) that

$$
\left\|\eta_{\alpha} w_{2, \alpha}\right\|_{H^{1}}^{2} \leq \int_{M}\left|\nabla \eta_{\alpha}\right|^{2} w_{2, \alpha}^{2} d v_{g}+C \mu_{\alpha}\left\|\eta_{\alpha} w_{2, \alpha}\right\|_{H^{1}} .
$$

By (4.37), since $\left|\nabla \eta_{\alpha}\right| \leq C r_{\alpha}^{-1}$, we get that

$$
\int_{M}\left|\nabla \eta_{\alpha}\right|^{2} w_{2, \alpha}^{2} d v_{g} \leq C r_{\alpha}^{2}\left(\frac{\mu_{\alpha}^{2}}{r_{\alpha}^{2}} \ln \left(\frac{r_{\alpha}}{\mu_{\alpha}}\right)\right)^{2}
$$

and by (4.9) it follows that

$$
\int_{M}\left|\nabla \eta_{\alpha}\right|^{2} w_{2, \alpha}^{2} d v_{g}=o\left(\mu_{\alpha}^{2} \ln ^{2} \frac{1}{\mu_{\alpha}}\right) .
$$

Coming back to (4.51), it follows that

$$
\left\|\eta_{\alpha} w_{2, \alpha}\right\|_{H^{1}}=o\left(\mu_{\alpha} \ln \frac{1}{\mu_{\alpha}}\right)
$$

By (4.44), (4.47) and (4.48), we then get with (4.52) that

$$
\begin{aligned}
& \int_{B_{x_{\alpha}}\left(r_{\alpha}\right)} v_{\alpha} u_{\alpha}^{2} d v_{g}=o\left(\mu_{\alpha}^{2} \ln \frac{1}{\mu_{\alpha}}\right) \text { and } \\
& \int_{B_{x_{\alpha}}\left(r_{\alpha}\right)} u_{\alpha} v_{\alpha}\left|X_{\alpha}\left(\nabla u_{\alpha}\right)\right| d v_{g}=o\left(\mu_{\alpha}^{2} \ln \frac{1}{\mu_{\alpha}}\right) .
\end{aligned}
$$

Coming back to (4.30) and (4.42) it follows that

$$
\mathcal{H}(0)=\frac{1}{64}\left(m_{0}^{2}-\omega^{2}-\frac{1}{6} S_{g}\left(x_{0}\right)\right) \lim _{\alpha \rightarrow+\infty} r_{\alpha}^{2} \ln \frac{r_{\alpha}}{\mu_{\alpha}} .
$$

By (4.54) we get that $\mathcal{H}(0) \leq 0$. At this point it remains to prove that $\rho_{\alpha}=O\left(r_{\alpha}\right)$. We prove that $\rho_{\alpha}=r_{\alpha}$. If not the case, then $r_{\alpha}<\rho_{\alpha}$ and we get with (4.11) that $(r \varphi(r))^{\prime}(1)=0$, where

$$
\begin{aligned}
\varphi(r) & =\frac{1}{\omega_{3} r^{3}} \int_{\partial B_{0}(r)} \tilde{u} d \sigma \\
& =\frac{8}{r^{2}}+\mathcal{H}(0) .
\end{aligned}
$$

Hence $\mathcal{H}(0)=8$ and we get a contradiction with $\mathcal{H}(0) \leq 0$. In other words, $\rho_{\alpha}=r_{\alpha}$ for all $\alpha \gg 1$. This ends the proof of the lemma.

Thanks to Lemma 4.2 we can now prove the uniform bounds in Theorem 0.3 This is the subject of what follows. 
Proof of the uniform bounds in Theorem 0.3. Let $(M, g)$ be a smooth compact Riemannian 4-dimensional manifold and $\left(\left(u_{\alpha}, v_{\alpha}\right)\right)_{\alpha}$ be a sequence of smooth positive solutions of (4.1) such that (0.7) holds true. By Druet, Hebey and Vétois 22 there exists $C>0$ such that for any $\alpha$ the following holds true: there exist $N_{\alpha} \in \mathbb{N}^{\star}$ and $N_{\alpha}$ critical points of $u_{\alpha}$, denoted by $\left(x_{1, \alpha}, x_{2, \alpha}, \ldots, x_{N_{\alpha}, \alpha}\right)$, such that

$$
d_{g}\left(x_{i, \alpha}, x_{j, \alpha}\right) u_{\alpha}\left(x_{i, \alpha}\right) \geq 1
$$

for all $i, j \in\left\{1, \ldots, N_{\alpha}\right\}, i \neq j$, and

$$
\left(\min _{i=1, \ldots, N_{\alpha}} d_{g}\left(x_{i, \alpha}, x\right)\right) u_{\alpha}(x) \leq C
$$

for all $x \in M$ and all $\alpha$. We define

$$
d_{\alpha}=\min _{1 \leq i<j \leq N_{\alpha}} d_{g}\left(x_{i, \alpha}, x_{j, \alpha}\right) .
$$

If $N_{\alpha}=1$, we set $d_{\alpha}=\frac{1}{4} i_{g}$, where $i_{g}$ is the injectivity radius of $(M, g)$. We claim that

$$
d_{\alpha} \nrightarrow \nrightarrow 0
$$

as $\alpha \rightarrow+\infty$. In order to prove this claim, we proceed by contradiction. Assuming on the contrary that $d_{\alpha} \rightarrow 0$ as $\alpha \rightarrow+\infty$, we see that $N_{\alpha} \geq 2$ for $\alpha$ large, and we can thus assume that the concentration points are ordered in such a way that

$$
d_{\alpha}=d_{g}\left(x_{1, \alpha}, x_{2, \alpha}\right) \leq d_{g}\left(x_{1, \alpha}, x_{3, \alpha}\right) \leq \cdots \leq d_{g}\left(x_{1, \alpha}, x_{N_{\alpha}, \alpha}\right) .
$$

We set, for $x \in B_{0}\left(\delta d_{\alpha}^{-1}\right), 0<\delta<\frac{1}{2} i_{g}$ fixed,

$$
\begin{aligned}
& \hat{u}_{\alpha}(x)=d_{\alpha} u_{\alpha}\left(\exp _{x_{1, \alpha}}\left(d_{\alpha} x\right)\right), \\
& \hat{h}_{\alpha}(x)=h_{\alpha}\left(\exp _{x_{1, \alpha}}\left(d_{\alpha} x\right)\right), \text { and } \\
& \hat{g}_{\alpha}(x)=\left(\exp _{x_{1, \alpha}}^{\star} g\right)\left(d_{\alpha} x\right) .
\end{aligned}
$$

It is clear that $\hat{g}_{\alpha} \rightarrow \xi$ in $C_{l o c}^{2}\left(\mathbb{R}^{n}\right)$ as $\alpha \rightarrow+\infty$ since $d_{\alpha} \rightarrow 0$ as $\alpha \rightarrow+\infty$. Thanks to (4.1) we have that

$$
\Delta_{\hat{g}_{\alpha}} \hat{u}_{\alpha}+d_{\alpha}^{2} \hat{h}_{\alpha} \hat{u}_{\alpha}=\hat{u}_{\alpha}^{3}
$$

in $B_{0}\left(\delta d_{\alpha}^{-1}\right)$, for all $i$. For any $R>0$, we also let $1 \leq N_{R, \alpha} \leq N_{\alpha}$ be such that

$$
\begin{aligned}
& d_{g}\left(x_{1, \alpha}, x_{i, \alpha}\right) \leq R d_{\alpha} \text { for } 1 \leq i \leq N_{R, \alpha}, \text { and } \\
& d_{g}\left(x_{1, \alpha}, x_{i, \alpha}\right)>R d_{\alpha} \text { for } N_{R, \alpha}+1 \leq i \leq N_{\alpha} .
\end{aligned}
$$

Such a $N_{R, \alpha}$ does exist thanks to (4.59). We also have that $N_{R, \alpha} \geq 2$ for all $R>1$ and that $\left(N_{R, \alpha}\right)_{\alpha}$ is uniformly bounded for all $R>0$ thanks to (4.57). In the sequel, we set

$$
\hat{x}_{i, \alpha}=d_{\alpha}^{-1} \exp _{x_{1, \alpha}}^{-1}\left(x_{i, \alpha}\right)
$$

for all $1 \leq i \leq N_{\alpha}$ such that $d_{g}\left(x_{1, \alpha}, x_{i, \alpha}\right) \leq \frac{1}{2} i_{g}$. Thanks to (4.56), for any $R>1$, there exists $C_{R}>0$ such that

$$
\sup _{B_{0}(R) \backslash \bigcup_{i=1}^{N_{R R, \alpha}} B_{\hat{x}_{i, \alpha}}\left(\frac{1}{R}\right)} \hat{u}_{\alpha} \leq C_{R} .
$$


By the Harnack inequality in Druet, Hebey and Vétois [22, for any $R>1$, there exists $D_{R}>1$ such that

$$
\left\|\nabla \hat{u}_{\alpha}\right\|_{L^{\infty}\left(\Omega_{R, \alpha}\right)} \leq D_{R} \sup _{\Omega_{R, \alpha}} \hat{u}_{\alpha} \leq D_{R}^{2} \inf _{\Omega_{R, \alpha}} \hat{u}_{\alpha}
$$

where

$$
\Omega_{R, \alpha}=B_{0}(R) \backslash \bigcup_{i=1}^{N_{2 R, \alpha}} B_{\hat{x}_{i, \alpha}}\left(\frac{1}{R}\right) .
$$

Assume first that, for some $R>0$, there exists $1 \leq i \leq N_{R, \alpha}$ such that

$$
\hat{u}_{\alpha}\left(\hat{x}_{i, \alpha}\right)=O(1) .
$$

The two first equations in (4.4) are satisfied by the sequences $x_{\alpha}=x_{i, \alpha}$ and $\rho_{\alpha}=$ $\frac{1}{8} d_{\alpha}$. Then it follows from (4.6) that the last equation in (4.4) cannot hold and thus that $\left(\hat{u}_{\alpha}\right)_{\alpha}$ is uniformly bounded in $B_{\hat{x}_{i, \alpha}}\left(\frac{3}{4}\right)$. In particular, by standard elliptic theory, and thanks to (4.60), $\left(\hat{u}_{\alpha}\right)_{\alpha}$ is uniformly bounded in $C^{1}\left(B_{\hat{x}_{i, \alpha}}\left(\frac{1}{2}\right)\right)$. Since, by (4.55), we have that

$$
\left|\hat{x}_{i, \alpha}\right|^{\frac{n-2}{2}}\left|\hat{u}_{\alpha}\left(\hat{x}_{i, \alpha}\right)\right| \geq 1,
$$

we get the existence of some $\delta_{i}>0$ such that

$$
\left|\hat{u}_{\alpha}\right| \geq \frac{1}{2}\left|\hat{x}_{i, \alpha}\right|^{1-\frac{n}{2}} \geq \frac{1}{2} R^{1-\frac{n}{2}}
$$

in $B_{\hat{x}_{i, \alpha}}\left(\delta_{i}\right)$. Assume now that, for some $R>0$, there exists $1 \leq i \leq N_{R, \alpha}$ such that

$$
\left|\hat{u}_{\alpha}\left(\hat{x}_{i, \alpha}\right)\right| \rightarrow+\infty
$$

as $\alpha \rightarrow+\infty$. Since (4.4) is satisfied by the sequences $x_{\alpha}=x_{i, \alpha}$ and $\rho_{\alpha}=\frac{1}{8} d_{\alpha}$, it follows from Lemma 4.2 that the sequence $\left(\left|\hat{u}_{\alpha}\left(\hat{x}_{i, \alpha}\right)\right| \times\left|\hat{u}_{\alpha}\right|\right)_{\alpha}$ is uniformly bounded in

$$
\hat{\Omega}_{\alpha}=B_{\hat{x}_{i, \alpha}}\left(\tilde{\delta}_{i}\right) \backslash B_{\hat{x}_{i, \alpha}}\left(\frac{\tilde{\delta}_{i}}{2}\right)
$$

for some $\tilde{\delta}_{i}>0$. Thus, using (4.62), we can deduce that these two situations are mutually exclusive in the sense that either (4.63) holds true for all $i$ or (4.64) holds true for all $i$. Now we split the conclusion of the proof into two cases.

In the first case we assume that there exist $R>0$ and $1 \leq i \leq N_{R, \alpha}$ such that $\hat{u}_{\alpha}\left(\hat{x}_{i, \alpha}\right)=O(1)$. Then, thanks to the above discussion, we get that $\hat{u}_{\alpha}\left(\hat{x}_{j, \alpha}\right)=O(1)$ for all $1 \leq j \leq N_{R, \alpha}$ and all $R>0$. As above, we get that $\left(\hat{u}_{\alpha}\right)_{\alpha}$ is uniformly bounded in $C_{l o c}^{1}\left(\mathbb{R}^{4}\right)$. Thus, by standard elliptic theory, there exists a subsequence of $\left(\hat{u}_{\alpha}\right)_{\alpha}$ which converges in $C_{l o c}^{1}\left(\mathbb{R}^{4}\right)$ to some $\hat{u}$ solution of $\Delta \hat{u}=\hat{u}^{3}$ in $\mathbb{R}^{4}$. By the above discussion, $|u|$ possesses at least two critical points, namely 0 and $\hat{x}_{2}$, the limit of $\hat{x}_{2, \alpha}$. This is absurd thanks to the classification of Caffarelli, Gidas and Spruck [14].

In the second case we assume that there exist $R>0$ and $1 \leq i \leq N_{R, \alpha}$ such that $\left|\hat{u}_{\alpha}\left(\hat{x}_{i, \alpha}\right)\right| \rightarrow+\infty$ as $\alpha \rightarrow+\infty$. Then, thanks to the above discussion, $\hat{u}_{\alpha}\left(\hat{x}_{j, \alpha}\right) \rightarrow$ $+\infty$ as $\alpha \rightarrow+\infty$, for all $1 \leq j \leq N_{R, \alpha}$ and all $R>0$. By (4.60) we have that

$$
\Delta_{\hat{g}_{\alpha}} \hat{v}_{\alpha}+d_{\alpha}^{2} \hat{h}_{\alpha} \hat{v}_{\alpha}=\frac{1}{\hat{u}_{\alpha}(0)^{2}} \hat{v}_{\alpha}^{3}
$$


where $\hat{v}_{\alpha}=\hat{u}_{\alpha}(0) \hat{u}_{\alpha}$. Applying Lemma 4.2 and standard elliptic theory, and thanks to (4.62) and to the above discussion, one easily checks that, after passing to a subsequence, $\hat{u}_{\alpha}(0) \hat{u}_{\alpha} \rightarrow \hat{G}$ in $C_{l o c}^{1}\left(\mathbb{R}^{n} \backslash\left\{\hat{x}_{i}\right\}_{i \in I}\right)$ as $\alpha \rightarrow+\infty$, where $I=\left\{1, \ldots, \lim _{R \rightarrow+\infty} \lim _{\alpha \rightarrow+\infty} N_{R, \alpha}\right\}$ and, for any $R>0$,

$$
\hat{G}(x)=\sum_{i=1}^{\tilde{N}_{R}} \frac{\Lambda_{i}}{\left|x-\hat{x}_{i}\right|^{2}}+\hat{H}_{R}(x)
$$

in $B_{0}(R)$, where $2 \leq \tilde{N}_{R} \leq N_{2 R}$ is such that $\left|\hat{x}_{\tilde{N}_{R}}\right| \leq R$ and $\left|\hat{x}_{\tilde{N}_{R}+1}\right|>R$, where $N_{2 R, \alpha} \rightarrow N_{2 R}$ as $\alpha \rightarrow+\infty$, where $\lambda_{i}>0$, and where $\hat{H}_{R}$ is a harmonic function in $B_{0}(R)$. Since $\hat{G} \geq 0$, we can write thanks to the maximum principle that, in a neighbourhood of the origin,

$$
\hat{G}(x)=\frac{\Lambda_{1}}{|x|^{n-2}}+\hat{H}(x),
$$

where $\hat{H}(0) \geq \Lambda_{2}-\Lambda_{1} R^{-2}-\Lambda_{2}(R-1)^{-2}$. Choosing $R$ large enough, we can ensure that $\hat{H}(0)>0$ and this is in contradiction with Lemma 4.2 ,

By the above discussion we get that (4.58) holds true. Clearly, this implies that $\left(N_{\alpha}\right)_{\alpha}$ is uniformly bounded. Let $\left(x_{\alpha}\right)_{\alpha}$ be a sequence of maximal points of $u_{\alpha}$. Thanks to (4.3) and to (4.58), we clearly have that (4.4) holds true for the sequences $\left(x_{\alpha}\right)_{\alpha}$ and $\rho_{\alpha}=\delta$ for some $\delta>0$ fixed. This clearly contradicts Lemma 4.2 and thus concludes the proof of the uniform bounds in Theorem 0.3

Existence and nonexistence of a priori estimates for critical elliptic Schrödinger type equations on manifolds have been investigated by Berti-Malchiodi [7, Brendle [8, 9, Brendle and Marques [10, Brézis and Li [11, Druet [15, 16, Druet and Hebey [17, 18, 19, Druet, Hebey, and Vétois [22, Druet and Laurain [23], Hebey [27, 28, Khuri, Marques and Schoen [30, Li and Zhang [32, 33, Li and Zhu 34, Marques [39, Micheletti, Pistoia and Vétois [40, Schoen [44, 45], and Vétois 48. In the subcritical case, a priori estimates for Schrödinger equations go back to the seminal work by Gidas and Spruck [24]. The above list is not exhaustive.

Acknowledgments: The first author was partially supported by the ANR grant ANR-08-BLAN-0335-01. The authors are indebted to Olivier Druet for a very valuable idea which has helped to improve the manuscript. They are also indebted to Valdimir Georgescu and Frédéric Robert for several interesting discussions on this work.

\section{REFERENCES}

[1] Adelberger, E., Dvali, G., and Gruzinov, A., Photon-Mass Bound Destroyed by Vortices, Phys. Rev. Lett., 98, 2007, 010402 (4pages).

[2] D'Aprile, T., and Wei, J., Layered solutions for a semilinear elliptic system in a ball, J. Differential Equations, 226, 269-294, 2006.

[3] Clustered solutions around harmonic centers to a coupled elliptic system, Ann. Inst. H. Poincaré Anal. Non Linéaire, 24, 605-628, 2007.

[4] Aubin, T., Equations différentielles non linéaires et problème de Yamabe concernant la courbure scalaire, J. Math. Pures Appl., 55, 269-296, 1976.

[5] Benci, V., and Fortunato, D., Solitary waves of the nonlinear Klein-Gordon field equation coupled with the Maxwell equations, Rev. Math. Phys., 14, 409-420, 2002.

[6] _ Spinning Q-balls for the Klein-Gordon-Maxwell equations, Commun. Math. Phys., To appear. 
[7] Berti, M., and Malchiodi, A., Non-compactness and multiplicity results for the Yamabe problem on $S^{n}$, J. Funct. Anal., 180, 210-241, 2001.

[8] Brendle, S., Blow-up phenomena for the Yamabe equation, J. Amer. Math. Soc., 21, 951-979, 2008.

[9] _ On the conformal scalar curvature equation and related problems, Surveys on Differential Geometry, Surveys in differential geometry. Vol. XII, 1-19, 2008.

[10] Brendle, S., and Marques, Blow-up phenomena for the Yamabe equation II, J. Differential Geom., 81, 225-250, 2009.

[11] Brézis, H., and Li, Y.Y., Some nonlinear elliptic equations have only constant solutions, $J$. Partial Differential Equations, 19, 208-217, 2006.

[12] Brézis, H., and Nirenberg, L., Positive solutions of nonlinear ellitpic equations involving critical Sobolev exponents, Comm. Pure Appl. Math., 36, 1983, 437-477.

[13] Byrne, J.C., Cosmic tests of Maxwell's equations. I - A photon rest mass, Astrophys. Space Sci., 46, 1977, 115-132.

[14] Caffarelli, L. A., Gidas, B., and Spruck, J., Asymptotic symmetry and local behavior of semilinear elliptic equations with critical Sobolev growth, Comm. Pure Appl. Math., 42, 271-297, 1989.

[15] Druet, O., From one bubble to several bubbles: The low-dimensional case, J. Differential Geom., 63, 399-473, 2003.

[16] Compactness for Yamabe metrics in low dimensions, Internat. Math. Res. Notices, 23, 1143-1191, 2004.

[17] Druet, O., and Hebey, E., Elliptic equations of Yamabe type, International Mathematics Research Surveys, 1, 1-113, 2005.

[18] Stability and instability for Einstein-scalar field Lichnerowicz equations on compact Riemannian manifolds, Math. Z., 263, 2009, 33-67.

[19] — Stability for strongly coupled critical elliptic systems in a fully inhomogeneous medium, Analysis and PDEs, 2, 2009, 305-359.

[20] _ Existence and a priori bounds for electrostatic Klein-Gordon-Maxwell systems in fully inhomogeneous spaces, Commun. Contemp. Math., To appear.

[21] Druet, O., Hebey, E., and Robert, F., Blow-up theory for elliptic PDEs in Riemannian geometry, Mathematical Notes, Princeton University Press, vol. 45, 2004.

[22] Druet, O., Hebey, E., and Vétois, J., Bounded stability for strongly coupled critical elliptic systems below the geometric threshold of the conformal Laplacian, J. Funct. Anal., to appear.

[23] Druet, O., and Laurain, P., Stability of the Pohozaev obstruction in dimension 3, J. Eur. Math. Soc., to appear.

[24] Gidas, B., and Spruck, J., A priori bounds for positive solutions of nonlinear elliptic equations, Comm. Part. Diff. Eq., 6, 883-901, 1981.

[25] Goldhaber, A.S., and Nieto, M.M., Terrestrial and Extraterrestrial limits on the photon mass, Rev. Mod. Phys., 43, 1971, 277-296.

[26] _ Photon and Graviton mass limits, Rev. Mod. Phys., 82, 2010, 939979.

[27] Hebey, E., Critical elliptic systems in potential form, Adv. Differential Equations, 11, 511$600,2006$.

[28] Diagonal compactness for critical elliptic systems in potential form, Comm. P.D.E., $32,1837-1881,2007$.

[29] Klainerman, S., and Tataru, D., On the optimal regularity for Yang-Mills equations in $\mathbb{R}^{4+1}$, J. Amer. Math. Soc., 12, 1999, 93-116.

[30] Khuri, M., Marques, F. C., and Schoen, R., A compactness theorem for the Yamabe Problem, J. Differential Geom., to appear.

[31] Li, Y.Y., and Zhang, L., Liouville-type theorems and Harnack-type inequalities for semilinear elliptic equations, J. Anal. Math., 90, 27-87, 2003.

[32] — A Harnack type inequality for the Yamabe equations in low dimensions, Calc. Var. Partial Differential Equations, 20, 133-151, 2004.

[33] Compactness of solutions to the Yamabe problem II, Calc. Var. PDE, 24, 185-237, 2005.

[34] - Yamabe type equations on three dimensional Riemannian manifolds, Commun. Contemp. Math., 1, 1-50, 1999.

[35] Long, E., Existence and stability of solitary waves in non-linear Klein-Gordon-Maxwell equations, Rev. Math. Phys., 18, 747-779, 2006. 
[36] Long, E., and Stuart, D., Effective dynamics for solitons in the nonlinear Klein Gordon Maxwell system and the Lorentz force law, Preprint, 2009.

[37] Luo,J., Gillies, G.T., and Tu, L.C., The mass of the photon, Rep. Prog. Phys., 68, 2005, $77-130$.

[38] Luo, J., and Tu, L.C., Experimental tests of Coulomb's Law and the photon rest mass, Metrologia, 41, 2004, S136-S146.

[39] Marques, F.C., A priori estimates for the Yamabe problem in the non-locally conformally flat case, J. Differential Geom., 71, 315-346, 2005.

[40] Micheletti, A.M., Pistoia, A., and Vétois, J., Blow-up solutions for asymptotically critical elliptic equations on Riemannian manifolds, Indiana Univ. Math. J., 58, 1719-1746, 2009.

[41] Pauli, W., Relativistic Field Theories of Elementary Particles, Rev. Mod. Phys., 13, 1941, 203-232.

[42] Rodnianski, I., and Tao, T., Global regularity for the Maxwell-Klein-Gordon equation with small critical Sobolev norm in high dimensions, Comm. Math. Phys., 251, 377-426, 2004.

[43] Ruegg, H., and Ruiz-Altaba, M., The Stueckelberg field, Int. J. Mod. Phys. A, 19, 2004, $3265-3348$.

[44] Schoen, R., Variational theory for the total scalar curvature functional for Riemannian metrics and related topics, in Topics in Calculus of Variations (Montecatini Terme, 1987), Lecture Notes in Math., vol. 1365, Springer-Verlag, Berlin, 120-154, 1989.

[45] - On the number of constant scalar curvature metrics in a conformal class, in Differential Geometry: A Symposium in Honor of Manfredo do Carmo, Proc. Int. Conf. (Rio de Janeiro, 1988). Pitman Monogr. Surveys Pure Appl. Math., vol. 52, Longman Sci. Tech., Harlow, 311-320, 1991.

[46] Schrödinger, E., The Earth's and the Sun's permanent magnetic fields in the unitary field theory, Proc. Roy. Irish Acad. A, 49, 1943, 135-148.

[47] Selberg, S., Almost optimal local well-posedness of the Klein-Gordon-Maxwell system in 1+4 dimensions, Comm. Part. Diff. Eq., 27, 2002, 1183-1227.

[48] Vétois, J., Multiple solutions for nonlinear elliptic equations on compact Riemannian manifolds, Internat. J. Math., 18, 1071-1111, 2007.

E. Hebey, Université de Cergy-Pontoise, Département de Mathématiques, Site de Saint-Martin, 2 avenue Adolphe Chauvin, 95302 Cergy-Pontoise cedex, France

E-mail address: Emmanuel.Hebey@math.u-cergy.fr

T.T. Truong, Université de Cergy-Pontoise, Département de Physique, Site de SaintMartin, 2 avenue Adolphe Chauvin, 95302 Cergy-Pontoise cedex, France

E-mail address: tuong.truong@u-cergy.fr 